\title{
The Formation of a Stable Sliding Clamp Discriminates MSH2-MSH3 and MSH2-MSH6 Mismatch Interaction
}

Brooke M. Britton ${ }^{1}$, James A. London ${ }^{1}$, Juana Martin-Lopez ${ }^{1}$, Nathan D. Jones ${ }^{1}$, Jiaquan Liu ${ }^{1}$, Jong-Bong Lee Le, $^{2,3}$ and Richard Fishel ${ }^{1^{*}}$

${ }^{1}$ Department of Cancer Biology and Genetics, The Ohio State University Wexner Medical Center, Columbus, $\mathrm{OH}, 43210$, USA

${ }^{2}$ Department of Physics, Pohang University of Science and Technology (POSTECH), Pohang, Korea

${ }^{3}$ Interdisciplinary Bioscience and Bioengineering, POSTECH, Pohang, Korea

* To whom correspondence should be addressed. Tel: +1-614-292-2484 Fax: +1-614-688-4994; Email: rfishel@osu.edu 


\section{ABSTRACT}

MutS homologs (MSH) are highly conserved core components of DNA mismatch repair (MMR). Mismatch recognition provokes ATP-binding by MSH proteins that drives a conformational transition from a short-lived lesion-searching clamp to an extremely stable sliding clamp on the DNA. Once on DNA the MSH sliding clamps provide a platform for the assembly of MMR strand-specific excision components beginning with the highly conserved MutL homologs (MLH/PMS). Previous studies with short mismatch-containing oligonucleotides revealed an MSH ATP hydrolysis (ATPase) cycle that included mismatch recognition, the formation of an ATP-bound sliding clamp and dissociation from the end of a mismatched DNA that ultimately recovers the mismatch binding conformation. We found that ATP-bound MSH complexes on blocked-end or very long DNA are extremely stable under a range of ionic conditions. These observations underpinned the development of a high-throughput fluorescence resonance energy transfer (FRET) system capable of clearly distinguishing between HsMSH2-HsMSH3 and HsMSH2-HsMSH6 activities that is suitable for chemical inhibitor screens.

\section{INTRODUCTION}

Mismatch repair (MMR) corrects polymerase misincorporation errors, selected chemical and/or physical DNA damage as well as sequence heterology resulting from DNA recombination (1). Homologs of the prototypical E.coli (Ec) EcMutS and EcMutL are the most highly conserved MMR components across biology. The function(s) of the MutS homologs (MSH) and MutL homologs (MLH/PMS) have been of interest for decades. It is well known that MSH proteins recognize mismatched nucleotides $(2,3)$. However, the operation of these essential components following mismatch recognition has been persistently unsettled $(1,4-6)$.

MSH proteins function as dimers or heterodimers with each half containing a highly conserved Walker A/B nucleotide-binding motif (7). In addition to simple nucleotide mismatches, 
MSH proteins recognize insertion-deletion loop-type (IDL) mismatches and several relatives have evolved to recognize recombination cross-over structures during meiosis (8-10). The MSH search and recognition process has been shown to involve the formation of an incipient clamp that interrogates the DNA by rotation coupled diffusion $(11,12)$. ATP binding by all MSH proteins results in the formation of a stable freely diffusing sliding clamp on the DNA $(8,10,12-17)$. The biochemical function of the MLH/PMS proteins had been largely enigmatic until recent single molecule imaging studies showed that the EcMutS sliding clamp provides a platform for EcMutL to form a second extremely stable sliding clamp on DNA containing a mismatch (18). In E.coli, these two sliding clamps function together and/or separately to enhance EcMutH DNA association and GATC-hemimethylation incision activity (18-20) as well as EcUvrD strand specific unwindingdisplacement activity (21). The human HsMSH2-HsMSH6 and HsMLH1-HsPMS2 similarly form a cascade of sliding clamps, although the detailed downstream functions remain poorly understood (22).

Mutation of HsMSH2, HsMSH6, HsMLH1, and HsPMS2 cause the most common cancer predisposition in humans known as Lynch syndrome or hereditary non-polyposis colorectal cancer (LS/HNPCC) $(23,24)$. This observation is consistent with a pathogenic dysfunction of the major HsMSH2-HsMSH6 or HsMLH1-HsPMS2 heterodimers. Most cells that contain these major human heterodimers also possess the HsMSH2-HsMSH3 heterodimer $(9,10)$. HsMSH2HsMSH3 primarily recognizes large IDL's, while HsMSH2-HsMSH6 recognizes a broad array of common replication errors that includes single nucleotide mismatches and small IDL's $(10,25)$. Mutations of HsMSH3 have not been found to cause LS/HNPCC (26-28). However, mutation of HsMSH3 appears to eliminate the pathogenic expansion of trinucleotide repeat (TNR) disease genes such as Huntington's disease and Myotonic dystrophy (29). Together, these observations suggest that hampering $\mathrm{HsMSH} 2-\mathrm{HsMSH} 3$ activity, without affecting HsMSH2-HsMSH6 functions, could engender a useful therapeutic for TNR disease pathologies while not encouraging cancer. 
Here, we have employed a variety of bulk and high-resolution biophysical methods to dissect MSH progression to a stable sliding clamp. We found that the mismatch binding peaked at relatively low ionic strengths, similar to previous studies $(13,16,30)$. The dissociation kinetics and lifetime of the ATP-bound MSH sliding clamp determined by surface plasmon resonance (SPR) analysis and single molecule total internal reflection fluorescence (smTIRF) microscopy, respectively, was unaffected over a wide range of ionic conditions. The extremely stable sliding clamp formed by MSH proteins on end-blocked DNA suggested that a fluorescence resonance energy transfer (FRET) system could be developed that was capable of clearly distinguishing HsMSH2-HsMSH3 and HsMSH2-HsMSH6 functions. We show that Cy5-labeled HsMSH2HsMSH3 uniquely forms sliding clamps with a Cy3-labeled blocked-end $(\mathrm{CA})_{4}$ IDL producing a strong time-averaged FRET signal. In contrast, Cy3-labeled HsMSH2-HsMHS6 appears more promiscuous but forms sliding clamps and time-averaged FRET more efficiently with Cy3-labeled DNA containing a G/T mismatch compared to the Cy3-labeled $(\mathrm{CA})_{4}$ IDL. This FRET system is extremely durable and can tolerate room temperature manipulations and DMSO concentrations below $0.5 \%$, a common solvent in high-throughput large-scale compound screens.

\section{RESULTS}

\section{lonic Dependence of Mismatch binding and ATP-induced Dissociation}

MSH proteins preferentially bind DNA containing mismatched nucleotides $(3,15)$. Previous electrophoretic mobility shift analysis (EMSA) showed that binding to both a mismatched and fully duplex DNA increased with decreasing ionic strength $(13,16,30)$. Similarly, kinetic analysis of $\mathrm{MSH}$-mismatched binding has been examined by surface plasmon resonance (SPR), which is capable of distinguishing the second-order association rate $\left(\mathrm{k}_{\mathrm{on}}\right)$ and the first-order dissociation rate $\left(\mathrm{k}_{\text {off }}\right)$ to generate equilibrium binding dissociation constants $\left(K_{D}=\mathrm{k}_{\text {off }} / \mathrm{k}_{\text {on }}\right)$ that are largely similar to $\operatorname{EMSA}(13,17,31)$. 
SPR was utilized to determine that the $K_{D}$ for EcMutS $(3.0 \pm 0.4 \mathrm{nM}$ and $1.5 \pm 0.4 \mathrm{nM})$ and HsMSH2-HsMSH6 $(0.7 \pm 0.3 \mathrm{nM}$ and $3.3 \pm 2.3 \mathrm{nM})$ on unblocked versus biotin-streptavidin blocked-end mismatched DNA, respectively (Supporting Fig. S1 and S2). These results confirm previous studies that demonstrated MSH binding to a mismatched was principally unaffected by whether the DNA end was open or blocked by a biotin-streptavidin linkage $(13,32)$.

We then examined the binding of EcMutS and HsMSH2-HsMSH6 to open and biotinstreptavidin blocked-end mismatched DNA by SPR over a range of ionic strength concentrations (Fig. 1; Table 1; Supporting Table S2). We observed very little variation in the $k_{\text {off }}$ for either open or blocked-end mismatched DNA (Table 1; Supporting Table S2). These results suggest that changes in mismatch binding $\left(K_{D}\right)$ by EcMutS and HsMSH2-HsMSH6 are mostly dependent on $k_{\text {on }}\left(K_{D} \sim \mathrm{C} / k_{\text {on }}\right.$, where $\mathrm{C}$ is a constant related to $\left.k_{\text {off }}\right)$. Unlike previous electrophoretic mobility shift analysis (EMSA) in which MSH mismatch binding continually increased with decreasing ionic strength, we found that the $k_{\text {on }}$ for EcMutS and HsMSH2-HsMSH6 peaked at $\sim 75 \mathrm{mM} \mathrm{NaCl}$ (Fig. 1) $(13,16,30)$. Calculating the apparent binding dissociation $K_{D \cdot a p p}\left(k_{\text {off }} / k_{o n}\right)$ did not change the shape of the curve since the $k_{\text {off }}$ increased by no more than by $20 \%$ at low $\mathrm{NaCl}$ concentrations, while the $k_{\text {on }}$ displayed more than a 2-fold change over the range of salt concentrations. These results appear to suggest that ionic strength influences $\mathrm{MSH}-$ mismatch binding differently when examined by the EMSA gel-based system compared to the SPR system that directly detects binding as surface mass changes.

We noted significant differences in the $k_{\text {on }}$ curves between open- and blocked-end mismatched DNA for both EcMutS and HsMSH2-HsMSH6, although the shapes appeared similar (Fig. 1). The largest difference occurred with EcMutS where the $k_{\text {on }}$ decreased as much as $\sim 2-$ fold with blocked-end mismatched DNA. These results appear to support anecdotal evidence that MutS homologs bind to oligonucleotide DNA ends when the mismatched DNA contains an open-end; a configuration that mimics double-stranded breaks (DSBs) which is rare in vivo (1). 
The addition of ATP to an MSH protein bound to a mismatch or lesion provokes the formation of a sliding clamp that dissociates from an open-ended DNA $(8,10,15,32)$. We examined the dissociation rate of EcMutS and HsMSH2-HsMSH6 bound to open- and blockedend mismatched DNA by SPR following the addition of ATP ( $k_{\text {off } \bullet A T P}$; Table 1 ; Supporting Table S2). As expected, the ATP-induced dissociation of EcMutS and HsMSH2-HsMSH6 from openended mismatched DNA was at least 10-fold faster than blocked-end mismatched DNA over a wide range of ionic strength conditions (Table 1; Supporting Table S2). These results are consistent with previous conclusions that ATP-bound MSH proteins rapidly dissociates from open DNA ends but are retained by blocked-end DNA $(13,14,32)$. We note that the dissociation of EcMutS and HsMSH2-HsMSH6 in the absence of ATP on both open- and blocked-end mismatched DNA ( $\left.k_{\text {off }}\right)$ appeared similar to the $k_{\text {off }}$ ATP with blocked-end mismatched DNA. While this rate equivalence may be a coincidence, it is possible that the dissociation mechanics of EcMutS and HsMSH2-HsMSH6 bound to a mismatch is similar to the dissociation mechanics of a trapped ATP-bound sliding clamp.

\section{ATP-bound MSH sliding clamps are extremely stable on mismatched DNA}

Single molecule total internal reflection fluorescence microscopy (smTIRF) was used to probe the stability of Alexa647-labeled EcMutS sliding clamps on mismatched DNA (Fig. 2). In this system the trajectory, diffusion dynamics and lifetime of single EcMutS sliding clamps were determined over a range of ionic strengths. Numerous single particles could be easily resolved on the mismatched DNA (Fig. 2A; Supporting Fig. S3) and the trajectories binned to determine the diffusion coefficient as well as the lifetime. We found that the diffusion coefficient increased with increasing ionic strength (Fig. 2B). These observations are consistent with previous studies of Thermus aquaticus (Taq) TaqMutS and suggest that EcMutS ATP-bound sliding clamps undergo thermal diffusion along the DNA while maintaining intermittent contact with the backbone (14). 
In contrast, the lifetime appeared relatively constant (195 $\pm 11 \mathrm{sec})$ suggesting that EcMutS sliding clamps remain stably linked to the mismatched DNA over a wide range of ionic strength (Fig. 2C;

\section{Supporting Fig. S4).}

We similarly examine the lifetime of Cy3-labeled HsMSH2-HsMSH3 and HsMSH2HsMSH6 sliding clamps on DNA by smTIRF (Fig. 2D and 2E, Methods). For these studies we used a G/T mismatch as the preferred substrate for HsMSH2-HsMSH6 and a CAG insertiondeletion loop-type (IDL) mismatch as the preferred substrate for HsMSH2-HsMSH3. While the lifetime of HsMSH2-HsMSH6 sliding clamps on DNA was similar to EcMutS $(163 \pm 40$ sec; Fig. 2D), the lifetime of HsMSH2-HsMSH3 was almost six-times longer (1315 \pm 389 sec; Fig. 2E). The analysis of HsMSH2-HsMSH3 was performed at different laser powers to assure photobleaching was not artificially reducing the lifetime (Fig. 2E, see colored histogram). Other than the IDL substrate preferences identified more than two decades ago (10), this is the first indication that $\mathrm{HsMSH} 2-\mathrm{HsMSH} 3$ may behave significantly different as a sliding clamp on the DNA compared to the major MSH proteins that are principally involved in post-replication MMR.

\section{A FRET-based system distinguishes human MSH sliding clamps}

The stability of MSH sliding clamps on a mismatched DNA suggested that a bulk fluorescence resonance energy transfer (FRET) scheme might rapidly distinguish mismatch bound and sliding clamp forms of the human MSH heterodimer proteins. This system is based on previous smTIRF studies that determined the binding and sliding clamp kinetics of the Thermus Aquaticus MutS (TaqMutS) (12). We designed a 41-mer oligonucleotide containing either no mismatch, a G/T mismatch or a $(\mathrm{CA})_{4}$ IDL with a biotin on both ends (Fig. 3A). These oligonucleotides were additionally labeled with Cy5 at a Thymine nucleotide nine base pairs from the mismatch (Fig. 3A). Previous studies with TaqMutS suggested that mispair-specific binding will place the Cy3MSH in close proximity to the Cy5-DNA resulting in relatively high FRET. Because the Cy3-label is located on the $\mathrm{N}$-terminus of $\mathrm{HsMSH} 2$ in the HsMSH2-HsMSH6 heterodimer and the $\mathrm{N}$ - 
terminus of HsMSH3 in the HsMSH2-HsMSH3 heterodimer, we expect a lower FRET efficiency than with the TaqMutS where the label was located on the clasp arm significantly closer to the Cy5-DNA label (12). In the presence of ATP, both HsMSH2-HsMSH3 and HsMSH2-HsMSH6 will form a sliding clamp $(32,33)$. However, when the glycosylation-free avidin derivative neutravidin is bound to the biotin ends of the DNA the ATP-bound MSH sliding clamps are projected to be retained where they will oscillate along the length of the oligonucleotide producing a timeaveraged intermediate FRET (Fig. 3A).

A fixed concentration of HsMSH2-HsMSH6 (20 nM) was examined in the presence of a 1:2 and 1:4 molar ratio of Cy5-labeled DNA (Fig. 3B and 3C). As a control, we found that HsMSH2-HsMSH6 only transiently interacts with the G/C duplex DNA, resulting in little or no FRET (Fig. 3B-E). In contrast, HsMSH2-HsMSH6 specifically binds to the oligonucleotide containing a G/T mismatch resulting in a statistically significant elevated FRET (Fig. 3B-E; G/C vs $\left.G / T, p_{40 n M}=0.0006, p_{80 n M}=0.0004\right)$. As predicted, the addition of ATP resulted in a FRET reduction at both DNA concentrations (Fig. 3B-E; G/T vs $G / T_{A T P}, p_{40 n M}=0.005, p_{80 n M}=0.03$ ), which remained significantly above the G/C background control (Fig. 3B-E; G/C vs G/T ATP, $_{40 n M}$ $=0.0007, p_{80 n M}=0.002$ ). We noted a slight reduction in the G/T-binding FRET efficiency of HsMSH2-HsMSH6 with increased DNA concentration that is likely due to an increased background of direct Cy5 excitation by the $532 \mathrm{~nm}$ laser.

The Saccharomyces cerevisiae (Sc) ScMSH2-ScMSH6 heterodimer is known to recognize small IDLs in addition to single nucleotide mismatches (34). We found that incubation of the $8 \mathrm{bp}(\mathrm{CA})_{4}$ IDL with HsMSH2-HsMSH6 resulted in significant FRET above the G/C control (Fig. 2B-E). Moreover, the FRET efficiency decreased in the presence of ATP consistent with the formation of a sliding clamp (Fig. 2B-E). In all cases, the FRET efficiency was reduced compared to the G/T mismatch (Fig. 2B-E). The observations are consistent with the conclusion that Cy3-labeled HsMSH2-HsMSH6 binds and forms an ATP-bound sliding clamp on Cy5-labeled 
mismatched DNA generating a strong and reproducible FRET. However, the FRET distinction between $\mathrm{G} / \mathrm{T}$ and $(\mathrm{CA})_{4}$ mismatched DNA by HsMSH2-HsMSH6 is limited.

Conversely, Cy3-labeled HsMSH2-HsMSH3 appears largely incapable of recognizing or forming sliding clamp with the G/T mismatched DNA resulting in FRET above the control G/C duplex DNA (Fig. 3A-D). Yet, a strong FRET was observed in the presence of the (CA) $)_{4}$ IDL mismatched oligonucleotide that resolved to a lower FRET in the presence of ATP (Fig. 3A-D). Compared to HsMSH2-HsMSH6 the distinction between G/T and (CA) $)_{4}$ IDL is significant. Moreover, the room temperature stability of HsMSH2-HsMSH3, where the smTIRF studies were performed, suggest that this FRET-based system might be useful as an initial inhibitorycompound screening tool.

\section{DMSO affects the FRET efficiency but is correctable.}

Most chemical compound libraries are dissolved in dimethyl sulfoxide (DMSO), a polar aprotic solvent capable of solubilizing polar and nonpolar compounds that is also miscible in water $(35,36)$. We examine the effect of DMSO on the protein and DNA fluorophore environment (Fig. 4). An intrinsic fluorescence increase was found around the FRET emission wavelengths (650$750 \mathrm{~nm}$; Fig. 4A). Moreover, 5\% DMSO significantly reduced the direct excitation and FRET excitation peaks when included with Cy3-labeled HsMSH2-HsMSH6 and Cy5-labeled G/T mismatched DNA. However, the decrease in Cy3 spectra appeared greater than the effect on the Cy5 spectra, which could dramatically affect the interpretation of FRET efficiency (Fig. 4B). We also noted a modest effect of DMSO on the direct excitation of Cy3-labeled HsMSH2HsMSH6 and HsMSH2-HsMSH3 (Fig. 4C,D). Nevertheless, the overall shape of the Cy3 emission curve appeared largely unchanged suggesting that FRET excitation of a Cy5 fluorophore is achievable.

The studies in the presence of 5\% DMSO (Fig. 4A,B) implied that any interpretation of FRET efficiency must be performed in comparison to FRET values obtained in the presence of a 
G/C duplex DNA. Moreover, DMSO concentrations above 1\% appeared to decrease the direct excitation of Cy3 significantly enough to reduce the FRET efficiency below detectable levels. Because HsMSH2-HsMSH6 displayed the lowest FRET efficiency compared to HsMSH2HsMSH3, we used it as a bellwether to examine the effect of DMSO on the ability to distinguish mismatch binding (elevated FRET) and sliding clamp formation (intermediate-low FRET; Fig. 5). The G/T-binding FRET efficiency of HsMSH2-HsMSH6 decreased by at least $50 \%$ as the DMSO concentration was increased to $1 \%$ (Fig. 5). Nevertheless, these FRET efficiencies remained statistically significant compared to the G/C duplex DNA substrate $\left(p_{40 \mathrm{nM}}=0.0001, \mathrm{p}_{80 \mathrm{nM}}=0.01\right)$. Similarly, in the presence of ATP the FRET efficiency of sliding clamps bound to the blocked-end G/T mismatched DNA also decreased with increasing DMSO (Fig. 5). However, at both $40 \mathrm{nM}$ and 80 nM DNA the FRET efficiency of a G/T mismatch in the presence of ATP was not significantly above the FRET efficiency of the G/C duplex DNA at concentrations of $0.5 \%$ DMSO and higher (Fig. 5). These observations are consistent with the conclusion that the MSH-DNA FRET-based system may be useful as an inhibitory-compound screening tool when the DMSO concentration is below $0.5 \%$. Perhaps more importantly, it appears that this FRET system may be capable of differentially distinguishing the effects of inhibitory-compounds on HsMSH2HsMSH6 and HsMSH2-HsMSH3 (discussed below).

\section{DISCUSSION}

Decades of studies have clearly determined that $\mathrm{MSH}$ proteins recognized mismatched nucleotides and in the presence of ATP form an exceptionally stable sliding clamp on the DNA $(8,10,12-14,16-18,32)$. This class of proteins includes members that appear principally involved in post-replication MMR as well as exclusive process such as those associated with double strand break (DSB) repair during meiosis $(1,37)$. In eukaryotes such as S.cerevisae and human cells, it is generally believed that two heterodimeric version, MSH2-MSH3 and MSH2-MSH6, play overlapping and redundant roles in post-replication MMR. However, it is worth noting that while 
MSH2-MSH6 appears quite promiscuous on recognizing all the single nucleotide mismatches as well as a variety of small-to-medium IDL mismatches $(15,38)$, MSH2-MSH3 only recognized single nucleotide mismatches poorly and clearly favors small-to-large IDL mismatches $(10,39)$. Moreover, the cellular concentration of MSH2-MSH3 appears to be at least 10-fold lower than MSH2-MSH6 $(26,40)$. Finally, no pathogenic mutations of HsMSH3 have been found that lead to LS/HNPCC, while mutations of the core post-replication MMR genes HsMSH2, HsMSH6, HsMLH1 and HsPMS2 have been identified as abundant causes of this cancer predisposition.

Using bulk biochemical and single molecule imaging analysis we have probed and compared the properties of E.coli EcMutS and human HsMSH2-HsMSH3 and HsMSH2-HsMSH6 proteins. All of these MSH proteins display exceptional stability as ATP-bound sliding clamps on DNA. Compared to TaqMutS (10 min), EcMutS (3.25 min) and HsMSH2-HsMSH6 (3.4 min) display a significantly shorter lifetime as sliding clamps on the mismatched DNA (Fig. 2C,E). However, it seems likely that the lifetime of TaqMutS might be inflated since ATP hydrolysis is required to release $\mathrm{MSH}$ sliding clamps from the DNA (12). Yet, Taq MutS is most active at temperatures above $40^{\circ} \mathrm{C}$, while the single molecule imaging studies that are essential to generate lifetime data are performed at room temperature $\left(\sim 23^{\circ} \mathrm{C}\right)$. Nevertheless, $\mathrm{HsMSH} 2-$ HsMSH3 displayed a sliding clamp lifetime on the mismatched DNA of 17 min (Fig. 2D). This observation was a significant surprise and was confirmed at two different laser power settings to eliminate photobleaching, which could contribute to an inaccurate lifetime. Moreover, we note that the single molecule imaging studies supporting this long lifetime included time points in excess of 60 min, suggesting that $\mathrm{HsMSH} 2-\mathrm{HsMSH} 3$ remains active for an exceedingly long time at room temperature $\left(\sim 23^{\circ} \mathrm{C}\right)$. Such protein stability is extremely important for inhibitory compound screening studies that utilize robotic distribution of components (see below). Taken as a whole, the extremely long lifetime of HsMSH2-HsMSH3 as a sliding clamp on DNA appears to widen the functional differences between it and the core MMR heterodimer HsMSH2-HsMSH6; potentially expanding and/or differentiating the cellular role(s) of HsMSH2-HsMSH3. 
A major difference with MSH2-MSH6 is the role of MSH2-MSH3 in TNR expansion that leads to devastating diseases such as Huntington's chorea and myotonic dystrophy (41). While mutation of the core MMR components that include MSH2-MSH6 results in expansions and contractions of short simple repeats (microsatellite instability) $(42,43)$, deletion of $M S H 3$ virtually eliminates TNR expansion $(44,45)$. These apparently opposing expansion-contraction phenotypes on repeat DNA sequences has never been adequately explained. One hypothesis for why HsMSH3 mutations have not been linked to a cancer phenotype contemplates that a defect in HsMSH3 will lead to a significant increase in frame-shift mutations. These frameshifts would likely result in large numbers of abnormal or truncated surface antigens that could stimulate an immune response resulting in a termination of $\mathrm{HsMSH} 3$ mutant cells before a cancer phenotype could be established. Supporting this notion is the observation that PD1 inhibitors that reactivate immune recognition cells (46) are exceedingly effective therapeutic treatments for MMR defective tumors where frameshift mutations remain a minor but highly immunogenic component of the total mutation load (47).

These observations suggested that the identification of specific inhibitors of HsMSH2HsMSH3 that have no effect on MSH2-MSH6 activities might be useful as therapeutics. Specific inhibition of HsMSH2-HsMSH3 could ameliorate the somatic effects that are generally the most immediately pathological in TNR diseases, while preventing the tumorigenesis associated with MMR defects. Our studies have reduced to practice a simple FRET-based screen that relies on the remarkably long lifetime and stability of MSH sliding clamps (Fig. 3 and 4). The assay relies on the conversion of mismatch binding events to a stable sliding clamp that continues to exhibit time-averaged FRET (Fig. 3A). We calculated the statistical effect size (z-factor) for highthroughput (HTP) drug screening comparing a variety of FRET-generating events (Supporting Fig. S5) $(48,49)$. What is most evident from these plots is that FRET events generated by HsMSH2-HsMSH3 provide the largest z-factor margin for the detection of inhibitory events. These calculations suggest that in initial HTP compound screen focused on HsMSH2-HsMSH3 
functions should provide an array of specific inhibitors. This initial screen could then be followed by a counter screen for HsMSH2-HsMSH6 functions to identify specific inhibitors of HsMSH2HsMSH3 that do not affect HsMSH2-HsMSH6. Because the z-factor for HsMSH2-HsMSH6 functions in significantly lower than $\mathrm{HsMSH} 2-\mathrm{HsMSH} 3$ functions, more redundant FRET repeats will be required to assure statistical significance (Supporting Fig. S5). Nevertheless, the stability of the FRET system should be able to easily accommodate the extra duplicates.

\section{EXPERIMENTAL PROCEDURES}

\section{EcMutS expression, purification, and labelling}

EcMutS protein was expressed, purified, and labeled with fluorophore as previously described (50). Briefly, EcMutS was expressed in BL21 AI E. coli cells and purified by FPLC using a NiNTA column (Qiagen) and Heparin (GE Healthcare) column (18). EcMutS-containing fractions were dialyzed in storage buffer (25 mM HEPES pH 7.8, 1 mM DTT, 0.1 mM EDTA, 150 mM NaCl, $20 \%$ glycerol) and frozen at $-80^{\circ} \mathrm{C}$.

For fluorophore labeled proteins, EcMutS and MtFGE (ratio 1:1) were dialyzed together in conversion buffer at $4^{\circ} \mathrm{C}$ for $48 \mathrm{hr}$ and then dialyzed in labeling buffer overnight. Proteins were then incubated with HIPS-AlexaFluor 647 at $0^{\circ} \mathrm{C}$ for $48 \mathrm{hr}(50)$. Labelled EcMutS was separated from free dye and MtFGE on a heparin column. Fractions were visualized on an 8\% SDS-PAGE and dialyzed in storage buffer (25 mM HEPES pH 7.8, 1 mM DTT, 0.1 mM EDTA, 150 mM NaCl, $20 \%$ glycerol) and frozen at $-80^{\circ} \mathrm{C}$.

\section{HsMSH2-HsMSH6 expression and purification}

HsMSH2-MSH6 protein was expressed and purified as previously described (51). Briefly, HsMSH2 and HsMSH6 were coexpressed in Hi5 insect cells using the Bac-to-Bac Baculovirus Expression System (Invitrogen). Protein was purified by FPLC using a Ni-NTA column (Qiagen), 
PBE-94 column (Sigma), HiLoad 16/60 Superdex 200 (GE Healthcare), and MonoQ 5/50 GL (GE Healthcare). HsMSH2-MSH6 containing fractions were dialyzed in storage buffer (25 mM HEPES $\mathrm{pH}$ 7.8, $1 \mathrm{mM}$ DTT, $0.1 \mathrm{mM}$ EDTA, $150 \mathrm{mM} \mathrm{NaCl}, 20 \%$ glycerol) and frozen at $-80^{\circ} \mathrm{C}$.

\section{DNA preparation}

For surface plasmon resonance DNAs (82-mers) were prepared as follows. Oligonucleotides were synthesized by Integrated DNA Technologies (Coralville, lowa; Supplementary Table S1). The single stranded (ss) oligos were purified by polyacrylamide gel electrophoresis (PAGE), and annealed overnight by step-down cooling in a thermocyler to create double stranded (ds) oligos. The G-Strand used in every construct contained the biotin moiety that is necessary for surface linkage (Supplementary Table S1). The complementary T-strand either contained a 5' digoxigenin (dig) modification or no modification (Supplementary Table S1) and when annealed to the G-strand created a G/T mismatch. The annealed oligos were purified using a GenPak Fax (Waters) column on an HPLC.

The DNA for smTIRF was prepared as previously described (18). Briefly, a 7-kb fragment of DNA was prepared by digesting a plasmid with Bsal. $\lambda$-phage DNA (3.2 nM, Thermo Scientific) was ligated with Lambda Mismatch 2A and Lambda Mismatch 2B (800 nM, Supplementary Table S1) followed by digestion with Bsal. The treated $\lambda$-DNA was then ligated with the 7-kb DNA, 1000x Lambda Hairpin Linker 1 and Lambda Hairpin Linker 2 (Supplementary Table S1). The 18.4-kb band was excised and purified utilizing $\beta$-agarase, ethanol precipitation and stored at $-80^{\circ} \mathrm{C}(18)$.

The 41-mer oligonucleotides for FRET studies were synthesized by Integrated DNA Technologies (Coralville, lowa) and double stranded oligos were prepared by annealing the GStrand with either the T-Strand (G/T mismatch) or the C-Strand (homoduplex; Supplementary Table S1). All strands contained 3' biotin moieties. The oligos were annealed overnight in a 
thermocycler by step-down cooling. The annealed oligos were purified from contaminates using a GenPak Fax column (Waters) by HPLC.

\section{Preparation of surface plasmon resonance (SPR) sensor chip}

A streptavidin coated Biacore 3000 SPR sensor chip (Sensor Chip SA, GE Healthcare) containing four channels was preconditioned with $\mathrm{NaOH}(50 \mathrm{mM})$. Biotin only and biotin-digoxigenin dsDNA (described above) was immobilized on the chip, with dsG/T-dig (blocked-end) DNA in channel 2 and dsG/T (open-end) DNA in channel 4, leaving channels 1 and 3 as buffer-only reference channels. The SPR response units (RUs) were kept within 10 between channels 2 and 4 to ensure the similar amounts of DNA was immobilized in each channel.

\section{SPR analysis of MSH activity}

The SPR flow scheme is outlined in Supplementary Figures S1 and S2. Briefly, the MSH protein was injected to monitor binding $\left(\mathrm{k}_{\mathrm{on}}\right)$ followed by a buffer wash step that monitored dissociation $\left(\mathrm{k}_{\text {off }}\right)$. The binding-dissociation was followed by injection of binding-dissociation buffer containing ATP that results in a transition to an $\mathrm{MSH}$ sliding clamp that rapidly dissociated from the open end of an unblocked mismatched DNA (koff॰ATP; Supplementary Figures S1B and S2B). In contrast, blocked-end mismatched DNA retains the MSH sliding clamps that exhibit a dissociation

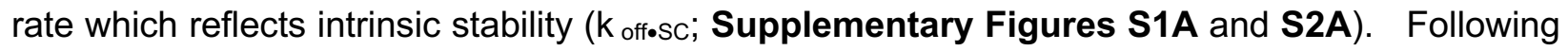
the ATP injection, the surface was regenerated with $1 \mathrm{M} \mathrm{NaCl}$ to remove any remaining protein while leaving the DNA undamaged.

For ionic strength binding/stability analysis, a titration of EcMutS and HsMSH2-MSH6 (0 - $100 \mathrm{nM}$ ) was first performed to determine the protein concentration that reflected $\sim 80 \%$ binding saturation. The experimental concentration for both $\mathrm{MSH}$ proteins (40 nM) for ionic strength binding/stability analysis fit to a Langmuir curve. Injections containing no protein were performed 
at the beginning and end of the analysis to ensure that background binding was unchanged between analysis.

\section{SPR analysis}

Experiments were performed at $25^{\circ} \mathrm{C}$. EcMutS and $\mathrm{HsMSH} 2-\mathrm{MSH} 6$ were examined independently using the same chip. A pre-blocking of the dig with anti-digoxigenin (anti-dig, 50 $\mathrm{nM}$, Roche) was performed prior to each experiment. While no change in SPR RUs was noted, previous work has shown that the anti-digoxigenin is enough to block/trap MSH clamps on the DNA $(51,52)$. The running buffer for both proteins was $25 \mathrm{mM}$ HEPES pH 7.8, $0.1 \mathrm{mM}$ DTT, 0.1 mM EDTA, $10 \mathrm{mM} \mathrm{MgCl} 2,200 \mu \mathrm{g} / \mathrm{ml}$ acetylated BSA (Promega), 0.01\% P-20 surfactant (GE Healthcare), $1 \mathrm{nM}$ anti-dig (Roche), and stated [ $\mathrm{NaCl}]$. Proteins were diluted prior to experiment to reduce glycerol and adjust $\mathrm{NaCl}$ concentrations. Binding experiments consisted of ten $\mathrm{NaCl}$ conditions $15,30,60,80,100,120,140,160,180,200 \mathrm{mM} \mathrm{NaCl}$. When ionic strength is referred to it is only that of $\mathrm{NaCl}$. RU changes reflect the binding and dissociation over time. ATP-induced dissociation was tested with an addition of running buffer containing ATP ( $1 \mathrm{mM}$, Roche) following binding of the MSH homolog.

Raw RUs were collected for each independent channel (1-4). Subtractions of the empty reference channels $(2-1,4-3)$ was performed to account of non-specific adsorption of protein to the surface. This subtraction data was used for all analysis. Data was normalized to the maximum of exponential fits for $\mathrm{MSH}$ binding. Runs were performed in triplicate. Association and dissociation constants were determined by fitting an exponential decay equation (Origin Labs) to the appropriate section of the curve.

\section{Preparation of smTIRF flow cell}

A laboratory engineered flow cell was used for all experiments consisting of a neutravidin coated, PEG passivated quartz slide surface. Laminar flow $(250 \mu \mathrm{L} / \mathrm{min})$ was used to inject and stretch 
the biotinylated dsDNA (as described above, $300 \mathrm{pM}$ ) in $300 \mu \mathrm{L}$ T-50 buffer $(20 \mathrm{mM}$ Tris-HCL, pH $7.5,50 \mathrm{mM} \mathrm{NaCl}$ ) on the surface. Prior to performing an experiment, the flow cells and all relevant tubing were prepared with BSA and P-20 to prevent nonspecific adsorption that may reduce concentrations.

\section{smTIRF experiments and data analysis}

Single molecule fluorescence data was attained on a home-built prism-type TIRF microscope (18) with green $(532 \mathrm{~nm})$ and red $(635 \mathrm{~nm})$ laser lines. Emissions were split by a Dual View optical setup (DV2, Photometrics) in bypass mode before collection using an EMCCD camera (ProEM Exelon512, Princeton Instruments). Laser excitation was modulated by the opening and closing of shutter controlled by Micro-Manager image capture software (53).

The single molecule imaging buffer was $20 \mathrm{mM}$ Tris- $\mathrm{HCl}(\mathrm{pH} 7.5), 5 \mathrm{mM} \mathrm{MgCl}, 0.1 \mathrm{mM}$ DTT, $200 \mu \mathrm{g} / \mathrm{ml}$ acetylated BSA (Promega), 0.0025\% P-20 surfactant (GE Healthcare), $1 \mathrm{mM}$ ATP (Roche), and stated [NaCl]. The imaging buffer included saturated ( 3 mM) Trolox, PCA (1 $\mathrm{mM})$, and PCD (10 $\mathrm{nM})$ to minimize photo blinking and photobleaching $(54,55)$.

EcMutS lifetime was calculated by flowing AF647-EcMutS (5 nM) into the prepared flow cell. In the absence of flow protein-DNA interactions were observed live. After recording Syto 59 (1000 nM, Invitrogen) was used to stain the DNA.

\section{Preparation of the Cy3-peptide}

$10 \mathrm{mg}$ of lyophilized peptide containing a Sortase recognition sequence(56) (57) and a single cystine residue for maleimide labeling (CLPETGG, GenScript) was dissolved in reaction buffer (50 mM Tris 7.0 and $5 \mathrm{mM} \mathrm{TCEP)} \mathrm{and} \mathrm{incubated} \mathrm{at} \mathrm{room} \mathrm{temperature} \mathrm{for} 30 \mathrm{~min}$. The reaction was then added to $3 \mathrm{mg}$ of lyophilized Sulfo-Cy3 maleimide (Lumiprobe) and incubated overnight at $4^{\circ} \mathrm{C}$. The labeled peptide was purified with reverse phase HPLC chromatography (Zorbax SB-C18, Agilent). Peak fractions were lyophilized and stored at $-80^{\circ} \mathrm{C}$ until needed. For 
labeling the peptide was dissolved in Sortase reaction buffer (25 mM HEPES pH 7.8, $150 \mathrm{mM}$ $\mathrm{NaCl}$ and $10 \%$ glycerol).

\section{MSH Sortase-tag addition, expression, purification, and labeling}

MSH proteins may be modified on the $\mathrm{N}$-terminus to contain a hexa-histidine (his 6 ), two serine spacers, a Sortase recognition sequence, and a flexible linker (GGGS) attached to the $\mathrm{MSH}$ protein of interest. For these studies the tags were placed on HsMSH6 and HsMSH3. MSH proteins were cloned into pFastBac1 (Invitrogen). The heterodimeric proteins (HsMSH2HsMSH6, HsMSH2-HsMSH3) were coexpressed as previously described $(10,25,32)$ in Sf9 cells using the Bac-to-Bac Baculovirus Expression System (Invitrogen). Protein was first purified by FPLC using a Ni-NTA column (Qiagen) followed by a heparin column (GE Healthcare). Peak fractions were pooled and combined with 4-times molar ratio of the Sortase protein and 50-times molar ratio of the Cy3-Peptide for $30 \mathrm{~min}$ at $4^{\circ} \mathrm{C}(56,58)$. The reaction is quenched with $20 \mathrm{mM}$ EDTA and loaded onto a spin-desalting column (40K Zeba Spin Desalting Column, Thermo Scientific). The eluent was then separated from the Cy3-peptide using a second heparin column (GE Healthcare) and polished using a MonoQ 5/50 GL (GE Healthcare). The HsMSH2-HsMSH6 and $\mathrm{HsMSH} 2-\mathrm{HsMSH} 3$ containing fractions were dialyzed in storage buffer (25 mM HEPES pH 7.8, $1 \mathrm{mM}$ DTT, $0.1 \mathrm{mM}$ EDTA, $150 \mathrm{mM} \mathrm{NaCl}, 20 \%$ glycerol) and frozen at $-80^{\circ} \mathrm{C}$.

\section{DNA fluorophore labeling and preparation}

DNA oligonucleotides are labeled with near $100 \%$ efficiency using fluorescent (Cy/AF) dyes. To increase homogeneity between multiple substrates, the G-strand is always labeled with the acceptor fluorophore and then annealed with a complementary strand containing no mismatch $(\mathrm{G} / \mathrm{C})$, a single nucleotide mismatch $(\mathrm{G} / \mathrm{T})$ or an IDL mismatch $\left[\mathrm{G} /+(\mathrm{CA})_{4}\right]$.

Single strand DNA containing an amino modifier C6 dT (IDT) in aqueous solution (10mM Tris- $\mathrm{HCl} \mathrm{pH}$ 8.0, 1mM EDTA) was precipitated using 3M sodium acetate $\mathrm{pH} 5.2,95 \%$ ethanol, 
and glycogen, followed by a wash with $70 \%$ ethanol to remove any impurities remaining from the synthesis process (Supplementary Table S1). The precipitate was then air-dried and resuspended in labeling buffer $(70-100 u l 0.1 \mathrm{M}$ sodium tetraborate $\mathrm{pH} 8.5)$. Cy-dye or analog, dissolved in dimethylformamide and added to the reaction in 10-30x molar excess. The reaction was mixed until completely dissolved and kept in foil rotating overnight at $23^{\circ} \mathrm{C}$ and $500 \mathrm{rpm}$. The overnight labeled material was ethanol precipitated following the same protocol as above twice to remove free dye. The final pellet should be fluorescent and if color is not observed the labeling should be repeated before subsequent HPLC purification steps. The final pellet was dissolved in triethylamine acetate (TEAA).

The fluorophore-labeled single-stranded DNA oligonucleotide was separated from unlabeled oligonucleotide and unincorporated free fluorophore by C18 reverse-phase HPLC chromatography (Poroshell 120 EC-C18, Agilent). Labeled fractions were pooled and ethanol precipitated as described above and resuspended in aqueous solution (10mM Tris- $\mathrm{HCl} \mathrm{pH} 8.0$, $1 \mathrm{mM}$ EDTA).

The fluorophore-labeled G-strand oligonucleotide was annealed with complementary

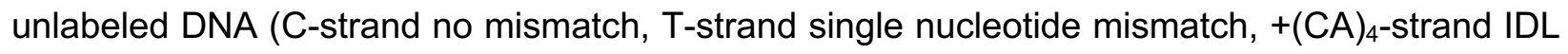
mismatch) in a $1: 1$ molar ratio by heating to $95^{\circ} \mathrm{C}$ and slow step cooling (Supplementary Table S1). The duplex DNA products were then purified from any remaining single stranded DNA substrates by ion exchange HPLC chromatography (Gen-Pack Fax, Waters). Duplex fractions were pooled, and ethanol precipitated as described above and resuspended in aqueous solution (10mM Tris- $\mathrm{HCl} \mathrm{pH} 8.0,1 \mathrm{mM}$ EDTA).

\section{FRET mismatch recognition assay analysis}

FRET detection was performed utilizing a FlouroMax-4 (Horiba Jobin Yvon) according to manufacturer's recommendations (59). The Flouromax-4 (Horiba Jobin Yvon) is a corrected photon counting system where the Intensity (counts per sec $/ \mu \mathrm{A}$ ) is: corrected signal detector 
(S1c) / corrected reference detector (R1c) or S1c/R1c. Because FRET calculations are ratios of acceptor and donor intensities, the S1c/R1c read-out from the FluoroMax (or any other corrected photon counting reader) may be used directly as measures of fluorescence intensity (I). While peak intensity at specific donor $(\sim 560 \mathrm{~nm})$ and acceptor $(\sim 670 \mathrm{~nm})$ wavelengths may be used as an initial screen for FRET efficiency calculations, increased accuracy is obtained by fitting the entire scanned peak intensities to a Gaussian function and integrating. Apparent FRET efficiency $\left(E_{A p p}\right)$ is calculated by: $E_{A p p}=\left(I_{A}-I_{A \cdot D N A}\right.$ Only $) /\left[\left(I_{A}-I_{A \cdot D N A}\right.\right.$ Only $)+\left(I_{D}-I_{D \cdot D N A}\right.$ Only $\left.)\right]$; where $I_{A}$ is the $\mathrm{MSH}+\mathrm{DNA}$ acceptor intensity, $\mathrm{I}_{\mathrm{A} \cdot \mathrm{DNA}}$ only is the acceptor intensity of the DNA alone, $\mathrm{I}_{\mathrm{D}}$ is the donor intensity, and ID.DNA Only is the donor intensity of the DNA alone. Thus, $E_{A p p}$ corrects for background contribution of $510 \mathrm{~nm}$ excitation that results in the acceptor Cy5-DNA Gaussian emissions with peaks at $\sim 560 \mathrm{~nm}$ and at $\sim 670 \mathrm{~nm}$ in the absence of donor Cy3-MSH protein. The donor Cy3MSH protein alone does not contribute to background Gaussian emission with a peak at $\sim 670$ $\mathrm{nm}$, and therefore does not require a correction factor.

The emission spectra was scanned from $545 \mathrm{~nm}$ to $720 \mathrm{~nm}$ following $510 \mathrm{~nm}$ excitation with a xenon lamp. The Cy3 peak was fit with 2 Gaussian functions due to the emission spectra of Cy3. The Cy5 emission peak was fit with a single Gaussian function. The $E_{\text {App }}$ FRET was calculated by measuring energy transfer under donor/acceptor intensity ratio (ratiometric FRET). A Cy5 labeled 41-bp duplex DNA containing a G/C duplex, G/T mismatch, or +(CA $)_{4}$ IDL mismatch with 3' biotin on both strands was end blocked with neutravidin was used. Cy3 labeled MSH protein was kept constant at $20 \mathrm{nM}$. The molar ratio was varied by increasing and decreasing the amount of DNA. Experiments were performed in a quartz cuvette on a FlouroMax-4 (Horiba Jobin Yvon) 5 minutes following the addition of protein. The addition of $1 \mathrm{mM}$ ATP was tested. Binding of $\mathrm{MSH}$ protein to the mismatch should result in a high FRET value (Figure 1, left). The addition of ATP should result in a sliding clamp that results in a time-averaged FRET (Figure 1, right). As the ratio of donor to acceptor is increased a higher FRET value is observed. 


\section{SUPPORTING INFORMATION}

Supporting Information are available online.

\section{AUTHOR CONTRIBURIONS}

B.M.B., J-B.L., and R.F. designed the experiments. B.M.B, J.L., J.M-L., and J.A.L. purified and labeled the proteins. B.M.B. purified the DNA substrates and performed surface plasmon resonance, single-molecule studies, and fluorimeter assays. B.M.B., N.D.J., J-B.L., and R.F. analyzed the data and wrote the paper. All authors participated in critical discussions.

\section{ACKNOWLEDGEMENTS}

We would like to thank members of the Fishel laboratory for insights and helpful discussions.

\section{FUNDING}

This work was supported by National Institutes of Health grants CA067007 and GM129764

(R.F.) and the Global Research Lab Program through the NRF of Korea funded by the Ministry of Science and ICT 2017K1A1A2013241 (J.-B.L.).

\section{REFERENCES}

\section{TABLE AND FIGURES LEGENDS}

Table 1. Dissociation Kinetics of EcMutS. Surface plasmon resonance dissociation studies. First-order rate constants of EcMutS $(40 \mathrm{nM})$ in the absence $\left(\mathrm{k}_{\text {off }}\right)$ and presence of ATP $(1 \mathrm{mM}$,

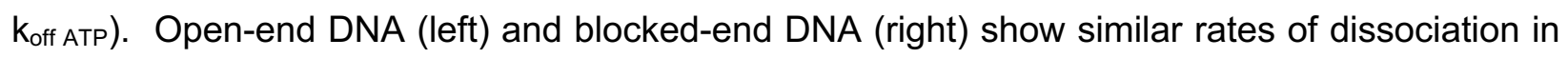
the absence of ATP ( $\left.\mathrm{k}_{\text {off }}\right)$. EcMutS sliding clamps quickly slide off open-end DNA (left), but are retained for ( 3 minutes) on blocked-end DNAs (right). 
Figure 1. Binding Affinity of EcMutS and HsMSH2-HsMSH6. Surface plasmon resonance association rates. Second-order rate constants $\left(k_{\text {on }}\right)$ of $(\mathbf{A})$ EcMutS (40 nM) and (B) HsMSH2HsMSH6 (40 nM) in the absence of ATP across a wide range of salt concentrations. Blockedend DNA is represented by black squares. Open-end DNA is represented by red squares. EcMutS and HsMSH2-HsMSH6 exhibit a peak binding affinity at $\sim 75 \mathrm{mM} \mathrm{NaCl}$. Both EcMutS and HsMSH2-HsMSH6 $k_{\text {on }}$ curves display the same shape, but EcMutS has a higher binding affinity toward open-end DNA, likely due to an end binding effect.

Figure 2. MSH Lifetimes. Single molecule analysis of individual labeled MSH sliding clamps. (A) Representative kymograph of EcMutS lifetime. Blue star indicates the mismatch position. (B) Box plots of diffusion for EcMutS $(5 \mathrm{nM})$ sliding clamps in the prescence of ATP $(1 \mathrm{mM})$ across a wide range of ionic strengths. Red line represents the mean, indent represents median. EcMutS sliding clamp diffusion rate increases with increasing $\mathrm{NaCl}$ concentration suggesting intermittent contact with the DNA backbone. (C)The lifetime of EcMutS $(5 \mathrm{nM})$ sliding clamps in the presence of ATP (1 mM) across a wide range of ionic strength conditions. Black square represents average value, whiskers represent standard deviation. Average lifetime was found to be $195 \pm 11$ seconds. (D) The lifetime of HsMSH2-HsMSH6 (1 nM) sliding clamps in the presence of ATP $(1 \mathrm{mM})$ at $100 \mathrm{mM} \mathrm{NaCl}$. Average lifetime was found to be $163 \pm 40$ seconds. (E) The lifetime of HsMSH2HsMSH3 (0.5 nM) sliding clamps in the presence of ATP (1 mM) at $100 \mathrm{mM} \mathrm{KGlu.} \mathrm{Average} \mathrm{lifetime}$ was found to be $1315 \pm 389$ seconds.

Figure 3. A FRET-Based Assay for Distinguishing HsMSH2-HsMSH6 and HsMSH2-HsMSH3 Activities. (A)The assay utilizes a short DNA oligo with double blocked-ends. These biotinneutravidin linkages will trap MSH clamps on the DNA and prevent dissociation from the end. A FRET acceptor (Cy5) has been placed on the DNA near the mismatch. A FRET donor (Cy3) has 
been placed on the protein of interest. MSH protein binding to a mismatch results in a high FRET state (top). Upon the addition of ATP the MSH clamps will transition into stable sliding clamps that diffuse along the DNA. This conformation of the protein results in an intermediate or time

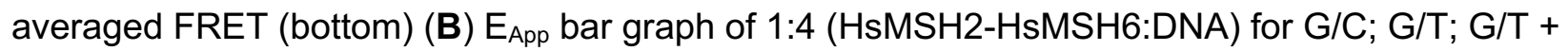
ATP;G/+(CA) 4 ; G/+(CA) 4 + ATP (mean \pm SD). HsMSH2-HsMSH6 does not bind G/C DNA (black) and preferentially binds G/T DNA (red). HsMSH2-HsMSH6 displays a reduced FRET efficiency upon binding ATP. ${ }^{* *}, \mathrm{p}<0.01 ; \mathrm{p}, 0.001$; by unpaired $t$ test. (C) Representative intensity vs. wavelength of 1:4 (HsMSH2-HsMSH6:DNA) for G/C; G/T; G/T + ATP; G/+(CA) $;$ G/+(CA) $)_{4}+$ ATP. (D) $E_{\text {App }}$ bar graph of 1:2 (HsMSH2-HsMSH6:DNA) for G/C; G/T; G/T + ATP; G/+(CA) $;$; G/+(CA) 4 + ATP (mean \pm SD). HsMSH2-HsMSH6 does not bind G/C DNA (black) and preferentially binds G/T DNA (red). HsMSH2-HsMSH6 displays a reduced FRET efficiency upon binding ATP. *, $\mathrm{p}<$ $0.05 ;{ }^{* *}, \mathrm{p}<0.01 ;{ }^{* *}, \mathrm{p}<0.001$; by unpaired $t$ test. (E) Representative intensity vs. wavelength of 1:2 (HsMSH2-HsMSH6:DNA) for G/C; G/T; G/T + ATP; G/+(CA) 4 ; G/+(CA) 4 + ATP.

Figure 4. FRET Analysis of Mismatch Recognition by HsMSH2-HsMSH3. (A) $E_{\text {App }}$ bar graph of 1:4 (HsMSH2-HsMSH3:DNA) for G/C; G/T; G/T + ATP; G/+(CA) $;$ G/+(CA) ${ }_{4}+$ ATP (mean \pm SD). HsMSH2-HsMSH3 does not bind G/C DNA (black) and preferentially binds G/+(CA) $)_{4}$ DNA (red). HsMSH2-HsMSH3 displays a reduced FRET efficiency upon binding ATP. ${ }^{* *}, \mathrm{p}<0.001$; by unpaired $t$ test. (B) Representative intensity vs. wavelength of 1:4 (HsMSH2-HsMSH3:DNA) for G/C; G/T; G/T + ATP; G/+(CA) $;$ G/+(CA) + ATP. (C) E $E_{A p p}$ bar graph of 1:2 (HsMSH2HsMSH3:DNA) for G/C; G/T; G/T + ATP; G/+(CA) $;$ G/+(CA) + ATP (mean \pm SD). HsMSH2HsMSH3 does not bind G/C DNA (black) and preferentially binds G/(CA) 4 DNA (red). HsMSH2HsMSH3 displays a reduced FRET efficiency upon binding ATP. ${ }^{* * *}, \mathrm{p}<0.001$; by unpaired $t$ test(D) Representative intensity vs. wavelength of 1:2 (HsMSH2-HsMSH6:DNA) for G/C; G/T; G/T + ATP; G/+(CA $)_{4} ; \mathrm{G} /+(\mathrm{CA})_{4}+$ ATP. 
Figure 5. The Effect of DMSO on Fluorescence Excitation and Emission. Representative intensity vs. wavelengths of $(\mathbf{A})$ DMSO in the absence of fluorophore. No effect is noted in the Cy3 spectra (550-650 nm). An increase in fluorescence is noted in the Cy5 spectra (650-750 $\mathrm{nm})$. (B) DMSO in the presence of fluorophore. A decrease in the Cy3 spectra is observed, while an increase in the Cy5 spectra occurs. This decrease in donor (Cy3) and increase in acceptor (Cy5) will result in a skewed FRET value. (C) DMSO effect on HsMSH2-HsMSH6. (D) DMSO effect on HsMSH2-HsMSH3.

Figure 6. The Effect of DMSO on HsMSH2-HsMSH6 FRET. (A) $E_{\text {App }}$ bar graph of 1:2 (HsMSH2HsMSH6:DNA) for G/C; G/T; G/T + ATP in the presence of DMSO (mean \pm SD). At 0.5\% DMSO and greater the ability to distinguish between the 3 states (unbound $(\mathrm{G} / \mathrm{C}$ ), bound to a mismatch $(\mathrm{G} / \mathrm{T})$, sliding clamp $(\mathrm{G} / \mathrm{T}+\mathrm{ATP}))$ is lost. *, $\mathrm{p}<0.05$; $^{* *}, \mathrm{p}<0.01$; ${ }^{* *}, \mathrm{p}<0.001$; by unpaired $t$ test. (B) $E_{\text {App }}$ bar graph of 1:4 (HsMSH2-HsMSH6:DNA) for G/C; G/T; G/T + ATP in the presence of DMSO (mean \pm SD). At $0.5 \%$ DMSO and greater the ability to distinguish between the 3 states (unbound $(\mathrm{G} / \mathrm{C})$, bound to a mismatch $(\mathrm{G} / \mathrm{T})$, sliding clamp $(\mathrm{G} / \mathrm{T}+\mathrm{ATP})$ ) is lost. ${ }^{*}, \mathrm{p}<0.05 ;{ }^{* *}, p$ $<0.01 ;{ }^{* * *}, \mathrm{p}<0.001$; by unpaired $t$ test.

1. Fishel, R. (2015) Mismatch repair. J Biol Chem 290, 26395-26403

2. Fishel, R. A., Siegel, E. C., and Kolodner, R. (1986) Gene conversion in Escherichia coli. Resolution of heteroallelic mismatched nucleotides by co-repair. J Mol Biol 188, 147-157

3. Su, S. S., and Modrich, P. (1986) Escherichia coli mutS-encoded protein binds to mismatched DNA base pairs. Proceedings of the National Academy of Sciences of the United States of America 83, 5057-5061

4. Kolodner, R. D., Mendillo, M. L., and Putnam, C. D. (2007) Coupling distant sites in DNA during DNA mismatch repair. Proceedings of the National Academy of Sciences of the United States of America 104, 12953-12954

5. Qiu, R., Sakato, M., Sacho, E. J., Wilkins, H., Zhang, X., Modrich, P., Hingorani, M. M., Erie, D. A., and Weninger, K. R. (2015) MutL traps MutS at a DNA mismatch. Proceedings of the National Academy of Sciences of the United States of America 112, 10914-10919

6. Ortega, J., Lee, G. S., Gu, L., Yang, W., and Li, G. M. (2021) Mispair-bound human MutSMutL complex triggers DNA incisions and activates mismatch repair. Cell Res 31, 542553 
7. Walker, J. E., Saraste, M., Runswick, M. J., and Gay, N. J. (1982) Distantly related sequences in the alpha- and beta-subunits of ATP synthase, myosin, kinases and other ATP-requiring enzymes and a common nucleotide binding fold. EMBO J 1, 945-951

8. Snowden, T., Acharya, S., Butz, C., Berardini, M., and Fishel, R. (2004) hMSH4-hMSH5 recognizes Holliday Junctions and forms a meiosis-specific sliding clamp that embraces homologous chromosomes. Mol Cell 15, 437-451

9. Palombo, F., laccarino, I., Nakajima, E., Ikejima, M., Shimada, T., and Jiricny, J. (1996) hMutSbeta, a heterodimer of hMSH2 and hMSH3, binds to insertion/deletion loops in DNA. Curr Biol 6, 1181-1184

10. Wilson, T., Guerrette, S., and Fishel, R. (1999) Dissociation of mismatch recognition and ATPase activity by hMSH2-hMSH3. J Biol Chem 274, 21659-21664

11. Gorman, J., Wang, F., Redding, S., Plys, A. J., Fazio, T., Wind, S., Alani, E. E., and Greene, E. C. (2012) Single-molecule imaging reveals target-search mechanisms during DNA mismatch repair. Proceedings of the National Academy of Sciences of the United States of America 109, E3074-3083

12. Jeong, C., Cho, W. K., Song, K. M., Cook, C., Yoon, T. Y., Ban, C., Fishel, R., and Lee, J. B. (2011) MutS switches between two fundamentally distinct clamps during mismatch repair. Nat Struct Mol Biol 18, 379-385

13. Acharya, S., Foster, P. L., Brooks, P., and Fishel, R. (2003) The coordinated functions of the E. coli MutS and MutL proteins in mismatch repair. Mol Cell 12, 233-246

14. Cho, W. K., Jeong, C., Kim, D., Chang, M., Song, K. M., Hanne, J., Ban, C., Fishel, R., and Lee, J. B. (2012) ATP alters the diffusion mechanics of MutS on mismatched DNA. Structure 20, 1264-1274

15. Gradia, S., Acharya, S., and Fishel, R. (1997) The human mismatch recognition complex hMSH2-hMSH6 functions as a novel molecular switch. Cell 91, 995-1005

16. Gradia, S., Acharya, S., and Fishel, R. (2000) The role of mismatched nucleotides in activating the hMSH2-hMSH6 molecular switch. J Biol Chem 275, 3922-3930

17. Mendillo, M. L., Mazur, D. J., and Kolodner, R. D. (2005) Analysis of the interaction between the Saccharomyces cerevisiae MSH2-MSH6 and MLH1-PMS1 complexes with DNA using a reversible DNA end-blocking system. J Biol Chem 280, 22245-22257

18. Liu, J., Hanne, J., Britton, B. M., Bennett, J., Kim, D., Lee, J. B., and Fishel, R. (2016) Cascading MutS and MutL sliding clamps control DNA diffusion to activate mismatch repair. Nature 539, 583-587

19. Hermans, N., Laffeber, C., Cristovão, M., Artola-Borán, M., Mardenborough, Y., Ikpa, P., Jaddoe, A., Winterwerp, H. H., Wyman, C., Jiricny, J., Kanaar, R., Friedhoff, P., and Lebbink, J. H. (2016) Dual daughter strand incision is processive and increases the efficiency of DNA mismatch repair. Nucleic Acids Res 44, 6770-6786

20. Mardenborough, Y. S. N., Nitsenko, K., Laffeber, C., Duboc, C., Sahin, E., Quessada-Vial, A., Winterwerp, H. H. K., Sixma, T. K., Kanaar, R., Friedhoff, P., Strick, T. R., and Lebbink, J. H. G. (2019) The unstructured linker arms of MutL enable GATC site incision beyond roadblocks during initiation of DNA mismatch repair. Nucleic Acids Res 47, 11667-11680

21. Liu, J., Lee, R., Britton, B. M., London, J. A., Yang, K., Hanne, J., Lee, J. B., and Fishel, R. (2019) MutL sliding clamps coordinate exonuclease-independent Escherichia coli mismatch repair. Nat Commun 10, 5294

22. London, J., Martín-López, J., Yang, I., Liu, J., Lee, J. B., and Fishel, R. (2021) Linker domain function predicts pathogenic MLH1 missense variants. Proceedings of the National Academy of Sciences of the United States of America 118

23. Fishel, R. (2015) Mismatch repair. Journal of Biological Chemistry 290, 26395-26403

24. Lynch, H. T., and Lynch, J. F. (1996) The Lynch Syndrome: Melding Natural History and Molecular Genetics to Genetic Counseling and Cancer Control. Cancer Control 3, 13-19 
25. Acharya, S., Wilson, T., Gradia, S., Kane, M. F., Guerrette, S., Marsischky, G. T., Kolodner, R., and Fishel, R. (1996) hMSH2 forms specific mispair-binding complexes with $\mathrm{hMSH} 3$ and hMSH6. Proceedings of the National Academy of Sciences of the United States of America 93, 13629-13634

26. Charbonneau, N., Amunugama, R., Schmutte, C., Yoder, K., and Fishel, R. (2009) Evidence that hMLH3 functions primarily in meiosis and in hMSH2-hMSH3 mismatch repair. Cancer Biol Ther 8, 1411-1420

27. Huang, J., Kuismanen, S. A., Liu, T., Chadwick, R. B., Johnson, C. K., Stevens, M. W., Richards, S. K., Meek, J. E., Gao, X., Wright, F. A., Mecklin, J. P., Järvinen, H. J., Grönberg, H., Bisgaard, M. L., Lindblom, A., and Peltomäki, P. (2001) MSH6 and MSH3 are rarely involved in genetic predisposition to nonpolypotic colon cancer. Cancer Res 61, 1619-1623

28. Watanabe, A., Ikejima, M., Suzuki, N., and Shimada, T. (1996) Genomic organization and expression of the human MSH3 gene. Genomics 31, 311-318

29. Jones, L., Houlden, H., and Tabrizi, S. J. (2017) DNA repair in the trinucleotide repeat disorders. Lancet Neurol 16, 88-96

30. Blackwell, L. J., Bjornson, K. P., and Modrich, P. (1998) DNA-dependent activation of the hMutSalpha ATPase. J Biol Chem 273, 32049-32054

31. Heinen, C. D., Wilson, T., Mazurek, A., Berardini, M., Butz, C., and Fishel, R. (2002) HNPCC mutations in hMSH2 result in reduced hMSH2-hMSH6 molecular switch functions. Cancer Cell 1, 469-478

32. Gradia, S., Subramanian, D., Wilson, T., Acharya, S., Makhov, A., Griffith, J., and Fishel, R. (1999) hMSH2-hMSH6 forms a hydrolysis-independent sliding clamp on mismatched DNA. Mol Cell 3, 255-261

33. Srivatsan, A., Bowen, N., and Kolodner, R. D. (2014) Mispair-specific recruitment of the Mlh1-Pms1 complex identifies repair substrates of the Saccharomyces cerevisiae Msh2Msh3 complex. J Biol Chem 289, 9352-9364

34. Marsischky, G. T., and Kolodner, R. D. (1999) Biochemical characterization of the interaction between the Saccharomyces cerevisiae MSH2-MSH6 complex and mispaired bases in DNA. J Biol Chem 274, 26668-26682

35. Balakin, K. V., Savchuk, N. P., and Tetko, I. V. (2006) In silico approaches to prediction of aqueous and DMSO solubility of drug-like compounds: trends, problems and solutions. Curr Med Chem 13, 223-241

36. Ilouga, P. E., Winkler, D., Kirchhoff, C., Schierholz, B., and Wölcke, J. (2007) Investigation of 3 industry-wide applied storage conditions for compound libraries. J Biomol Screen 12 , 21-32

37. Spies, M., and Fishel, R. (2015) Mismatch repair during homologous and homeologous recombination. Cold Spring Harb Perspect Biol 7, a022657

38. Marsischky, G. T., Filosi, N., Kane, M. F., and Kolodner, R. (1996) Redundancy of Saccharomyces cerevisiae $\mathrm{MSH} 3$ and $\mathrm{MSH} 6$ in $\mathrm{MSH} 2-$ dependent mismatch repair. Genes Dev 10, 407-420

39. Kolodner, R. D., and Marsischky, G. T. (1999) Eukaryotic DNA mismatch repair. Curr Opin Genet Dev 9, 89-96

40. Marra, G., laccarino, I., Lettieri, T., Roscilli, G., Delmastro, P., and Jiricny, J. (1998) Mismatch repair deficiency associated with overexpression of the MSH3 gene. Proceedings of the National Academy of Sciences of the United States of America 95, 8568-8573

41. Pearson, C. E., and Sinden, R. R. (1998) Trinucleotide repeat DNA structures: dynamic mutations from dynamic DNA. Curr Opin Struct Biol 8, 321-330 
42. Martín-López, J. V., Barrios, Y., Medina-Arana, V., Andújar, M., Lee, S., Gu, L., Li, G. M., Rüschoff, J., Salido, E., and Fishel, R. (2012) The hMSH2(M688R) Lynch syndrome mutation may function as a dominant negative. Carcinogenesis 33, 1647-1654

43. Martín-López, J. V., and Fishel, R. (2013) The mechanism of mismatch repair and the functional analysis of mismatch repair defects in Lynch syndrome. Fam Cancer 12, 159168

44. Kantartzis, A., Williams, G. M., Balakrishnan, L., Roberts, R. L., Surtees, J. A., and Bambara, R. A. (2012) Msh2-Msh3 interferes with Okazaki fragment processing to promote trinucleotide repeat expansions. Cell Rep 2, 216-222

45. Williams, G. M., and Surtees, J. A. (2015) MSH3 Promotes Dynamic Behavior of Trinucleotide Repeat Tracts In Vivo. Genetics 200, 737-754

46. Topalian, S. L., Drake, C. G., and Pardoll, D. M. (2015) Immune checkpoint blockade: a common denominator approach to cancer therapy. Cancer Cell 27, 450-461

47. Le, D. T., Durham, J. N., Smith, K. N., Wang, H., Bartlett, B. R., Aulakh, L. K., Lu, S., Kemberling, H., Wilt, C., Luber, B. S., Wong, F., Azad, N. S., Rucki, A. A., Laheru, D., Donehower, R., Zaheer, A., Fisher, G. A., Crocenzi, T. S., Lee, J. J., Greten, T. F., Duffy, A. G., Ciombor, K. K., Eyring, A. D., Lam, B. H., Joe, A., Kang, S. P., Holdhoff, M., Danilova, L., Cope, L., Meyer, C., Zhou, S., Goldberg, R. M., Armstrong, D. K., Bever, K. M., Fader, A. N., Taube, J., Housseau, F., Spetzler, D., Xiao, N., Pardoll, D. M., Papadopoulos, N., Kinzler, K. W., Eshleman, J. R., Vogelstein, B., Anders, R. A., and Diaz, L. A., Jr. (2017) Mismatch repair deficiency predicts response of solid tumors to PD1 blockade. Science 357, 409-413

48. Zhang, J. H., Chung, T. D., and Oldenburg, K. R. (1999) A Simple Statistical Parameter for Use in Evaluation and Validation of High Throughput Screening Assays. J Biomol Screen 4, 67-73

49. Sui, Y., and Wu, Z. (2007) Alternative statistical parameter for high-throughput screening assay quality assessment. J Biomol Screen 12, 229-234

50. Liu, J., Hanne, J., Britton, B. M., Shoffner, M., Albers, A. E., Bennett, J., Zatezalo, R., Barfield, R., Rabuka, D., Lee, J. B., and Fishel, R. (2015) An Efficient Site-Specific Method for Irreversible Covalent Labeling of Proteins with a Fluorophore. Scientific Reports 5

51. Heinen, C. D., Cyr, J. L., Cook, C., Punja, N., Sakato, M., Forties, R. A., Lopez, J. M., Hingorani, M. M., and Fishel, R. (2011) Human MSH2 (hMSH2) protein controls ATP processing by hMSH2-hMSH6. Journal of Biological Chemistry 286, 40287-40295

52. Hanne, J., Britton, B. M., Park, J., Liu, J., Martin-Lopez, J., Jones, N., Schoffner, M., Klajner, P., Bundschuh, R., Lee, J. B., and Fishel, R. (2018) MutS homolog sliding clamps shield the DNA from binding proteins. J Biol Chem 293, 14285-14294

53. Edelstein, A., Amodaj, N., Hoover, K., Vale, R., and Stuurman, N. (2010) Computer control of microscopes using manager. Current Protocols in Molecular Biology, 14.20.11-14.20.17

54. Senavirathne, G., Liu, J., Lopez, M. A., Jr., Hanne, J., Martin-Lopez, J., Lee, J. B., Yoder, K. E., and Fishel, R. (2015) Widespread nuclease contamination in commonly used oxygen-scavenging systems. Nature Methods 12, 901-902

55. Senavirathne, G., Lopez, M. A., Jr., Messer, R., Fishel, R., and Yoder, K. E. (2018) Expression and purification of nuclease-free protocatechuate 3,4-dioxygenase for prolonged single-molecule fluorescence imaging. Anal Biochem 556, 78-84

56. Popp, M. W., Antos, J. M., and Ploegh, H. L. (2009) Site-specific protein labeling via sortase-mediated transpeptidation. Curr Protoc Protein Sci Chapter 15, Unit 15.13

57. Dorr, B. M., Ham, H. O., An, C., Chaikof, E. L., and Liu, D. R. (2014) Reprogramming the specificity of sortase enzymes. Proceedings of the National Academy of Sciences of the United States of America 111, 13343-13348 
bioRxiv preprint doi: https://doi.org/10.1101/2021.10.21.465318; this version posted October 21, 2021. The copyright holder for this preprint

(which was not certified by peer review) is the author/funder, who has granted bioRxiv a license to display the preprint in perpetuity. It is made available under aCC-BY-NC-ND 4.0 International license.

58. Antos, J. M., Chew, G. L., Guimaraes, C. P., Yoder, N. C., Grotenbreg, G. M., Popp, M. W., and Ploegh, H. L. (2009) Site-specific N- and C-terminal labeling of a single polypeptide using sortases of different specificity. J Am Chem Soc 131, 10800-10801

59. Melhuish, W. H. (1972) Absolute Spectrofluorometry. J Res Natl Bur Stand A Phys Chem 76a, 547-560 
bioRxiv preprint doi: https://doi.org/10.1101/2021.10.21.465318; this version posted October 21, 2021. The copyright holder for this preprint (which was not certified by peer review) is the author/funder, who has granted bioRxiv a license to display the preprint in perpetuity. It is made available under aCC-BY-NC-ND 4.0 International license.

Figure 1

A

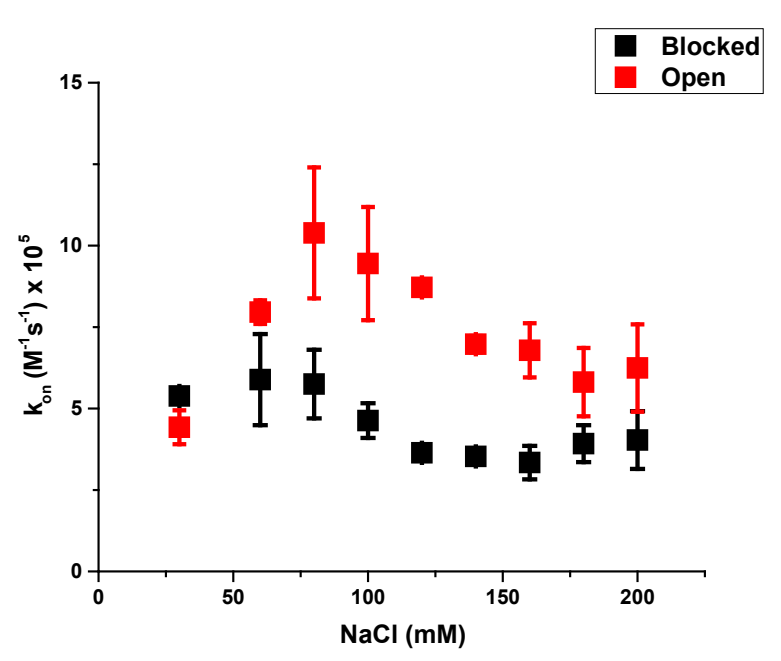

B

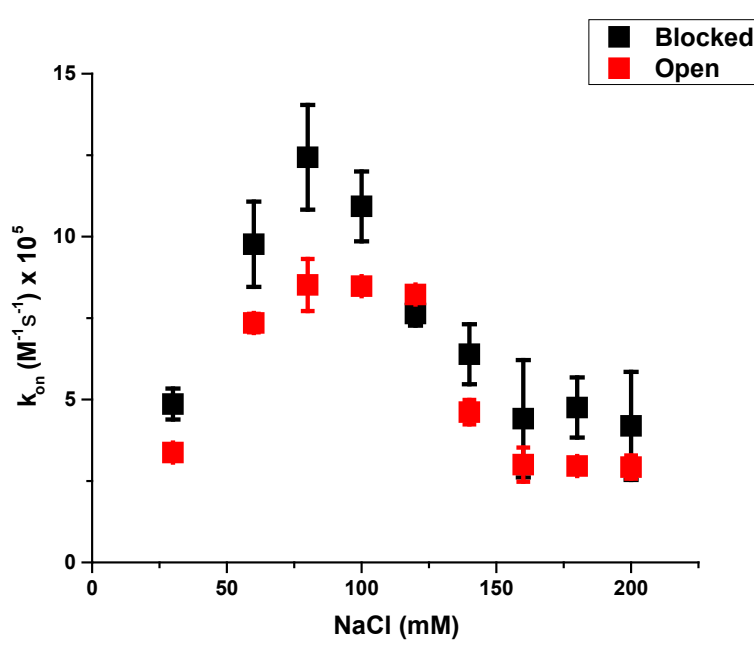


Figure 2

A

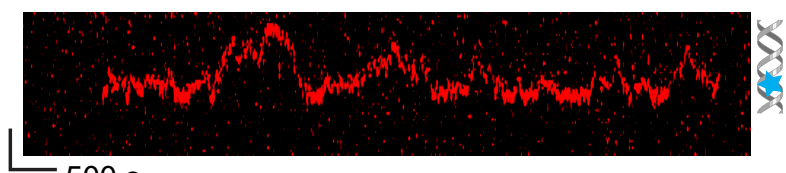

$500 \mathrm{~s}$

B

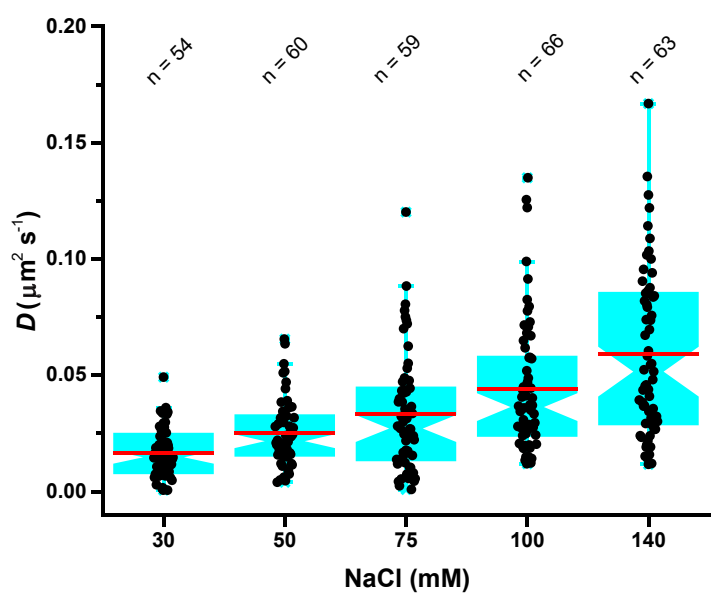

D

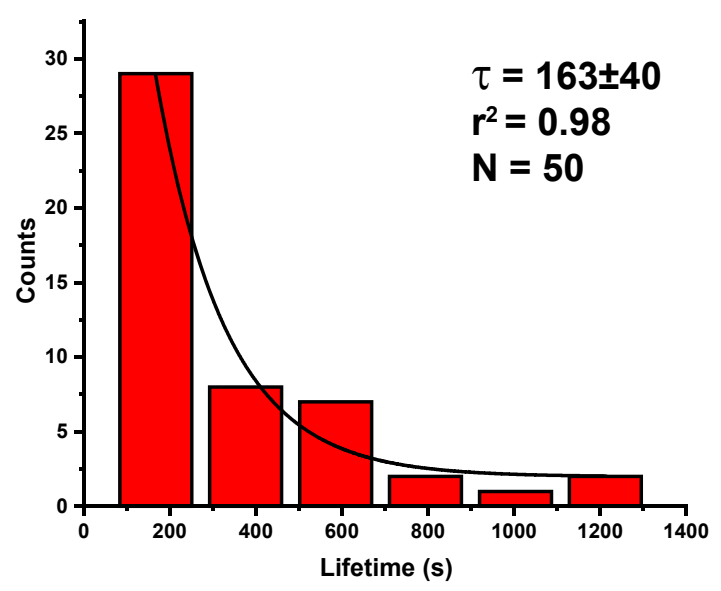

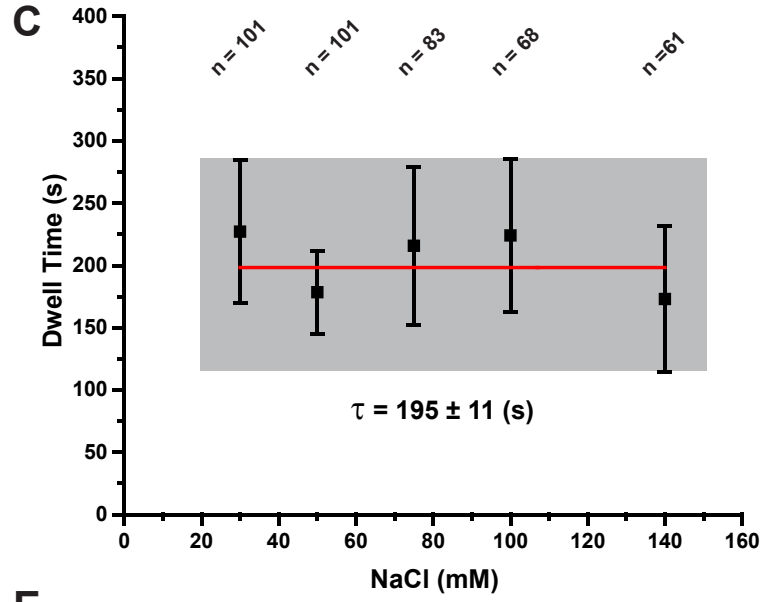

$\mathbf{E}$

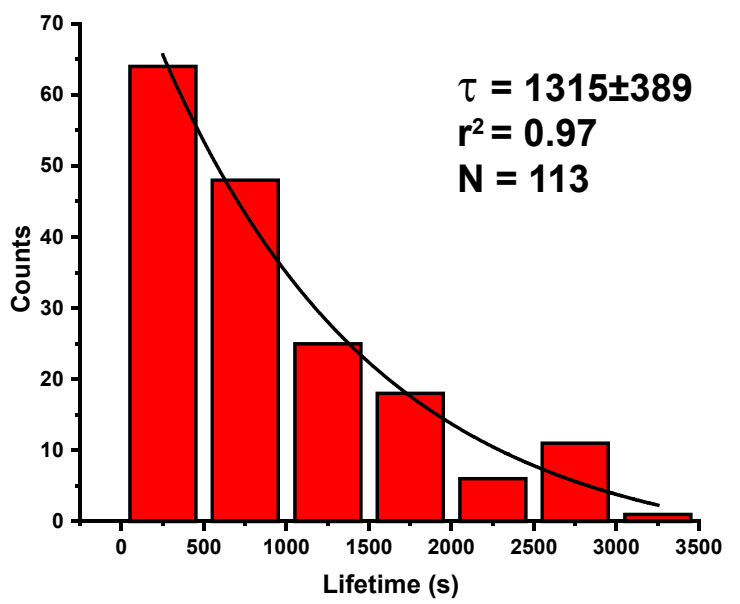


Figure 3

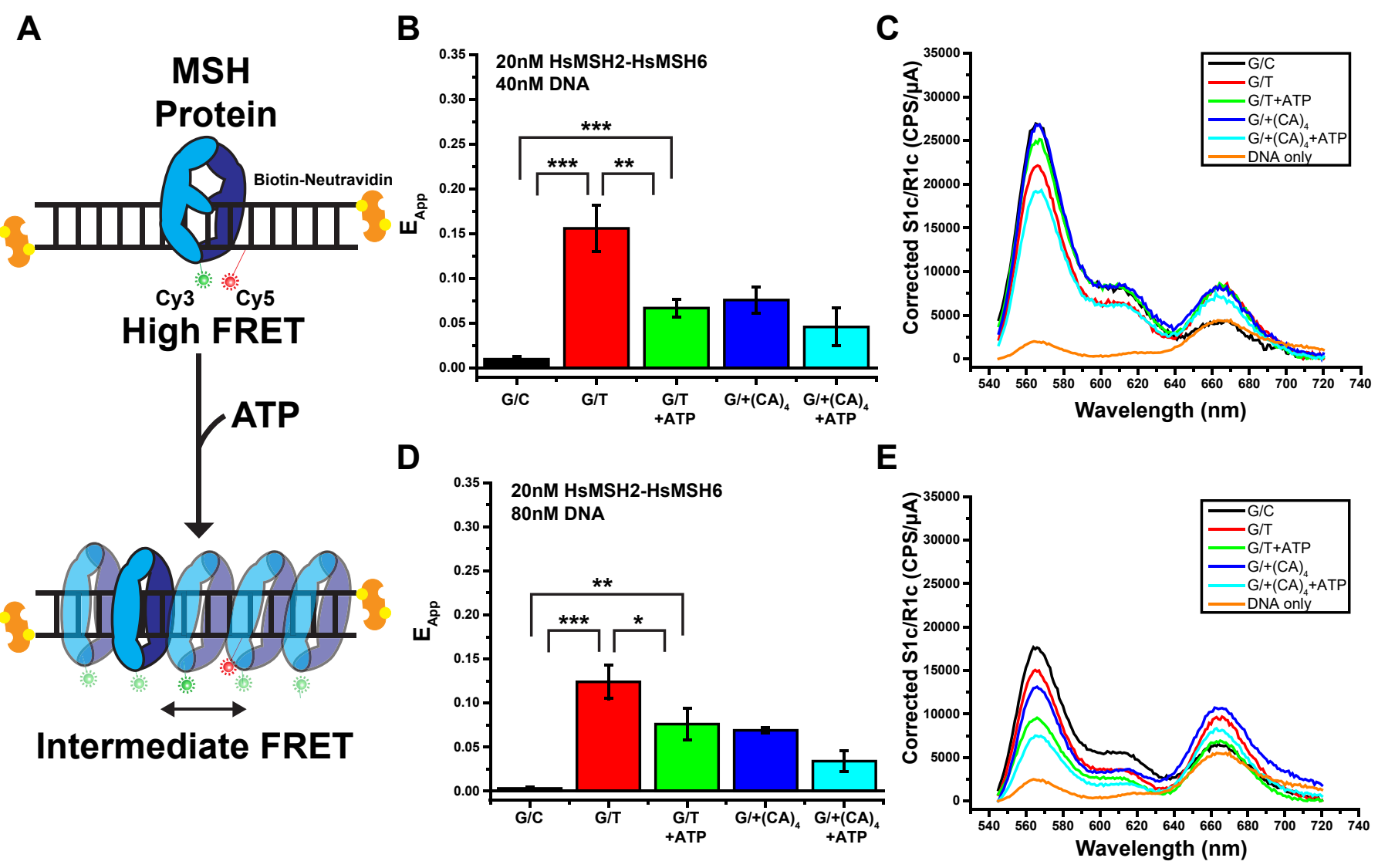


bioRxiv preprint doi: https://doi.org/10.1101/2021.1021.465318; this version posted October 21, 2021. The copyright holder for this preprint (which was not certified by peer review) is the author/funder, who has granted bioRxiv a license to display the preprint in perpetuity. It is made available under aCC-BY-NC-ND 4.0 International license.

Figure 4

A

20nM HsMSH2-HsMSH3 40nM DNA

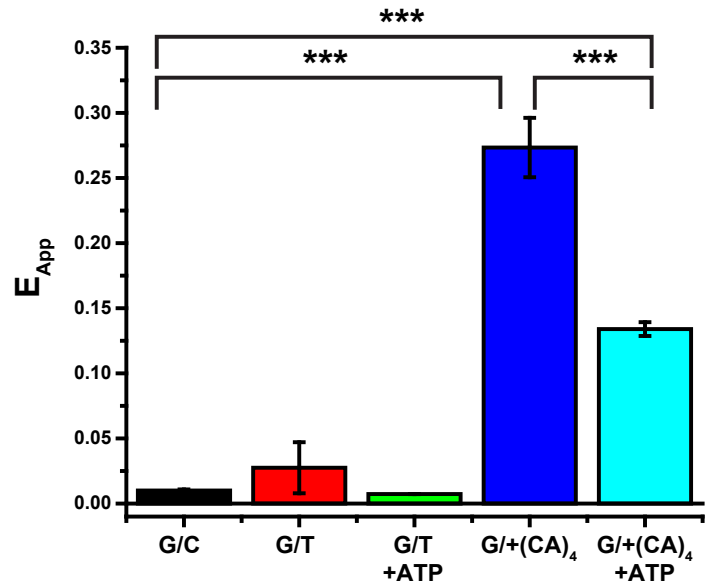

C 20nM HsMSH2-HsMSH3 80nM DNA

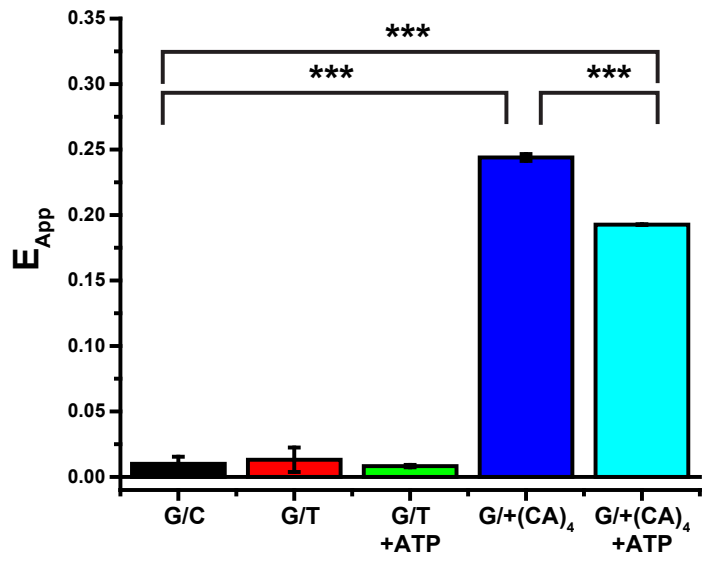

B

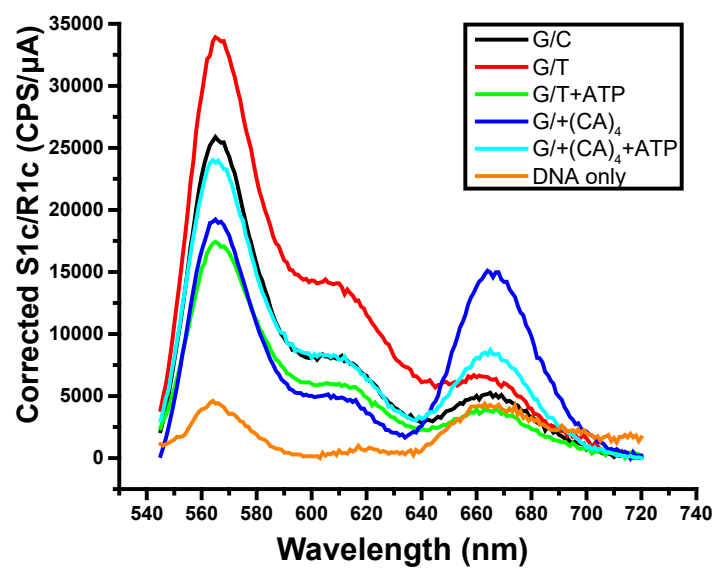

D

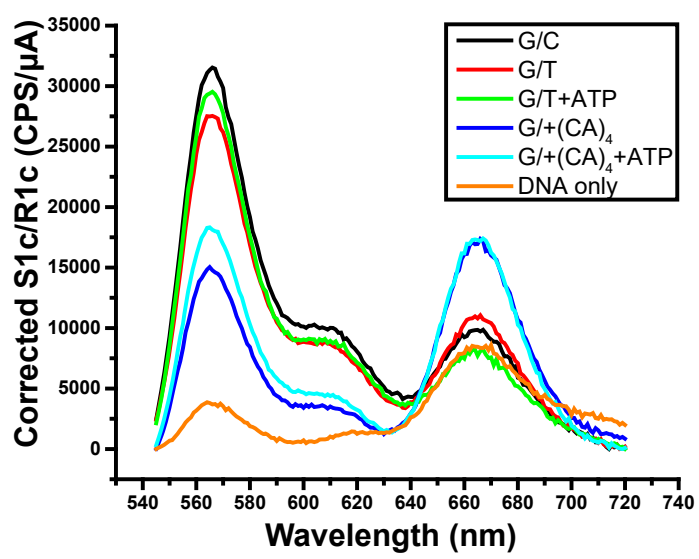


A

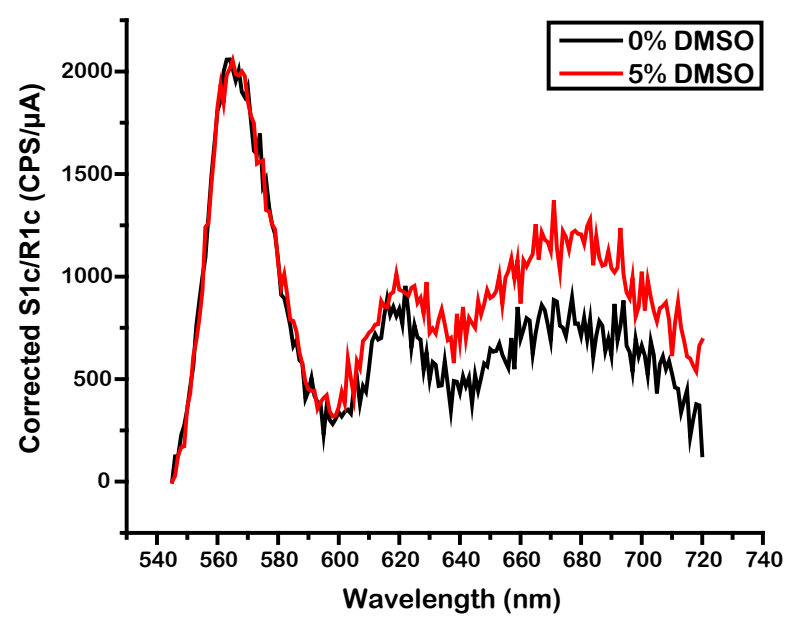

C

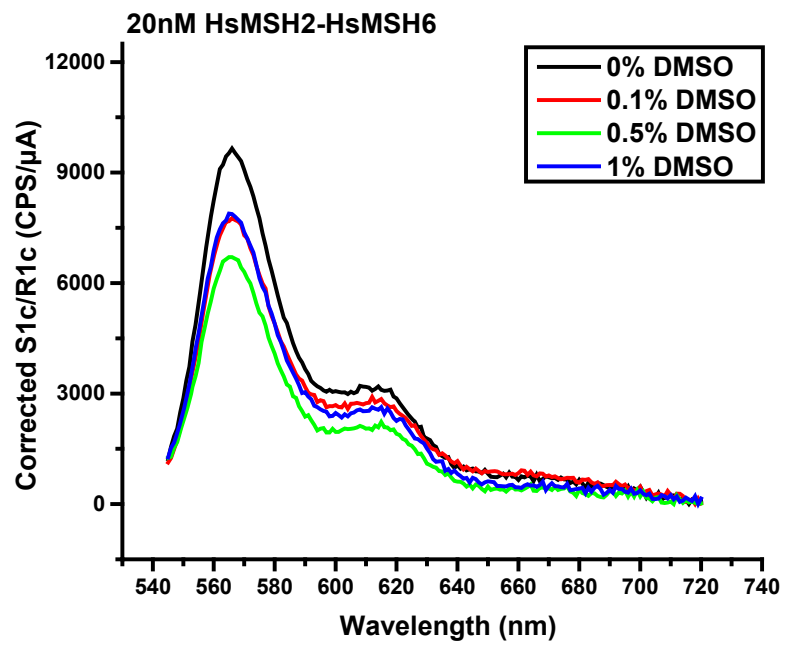

B

20nM HsMSH2-HsMSH6 80nM DNA

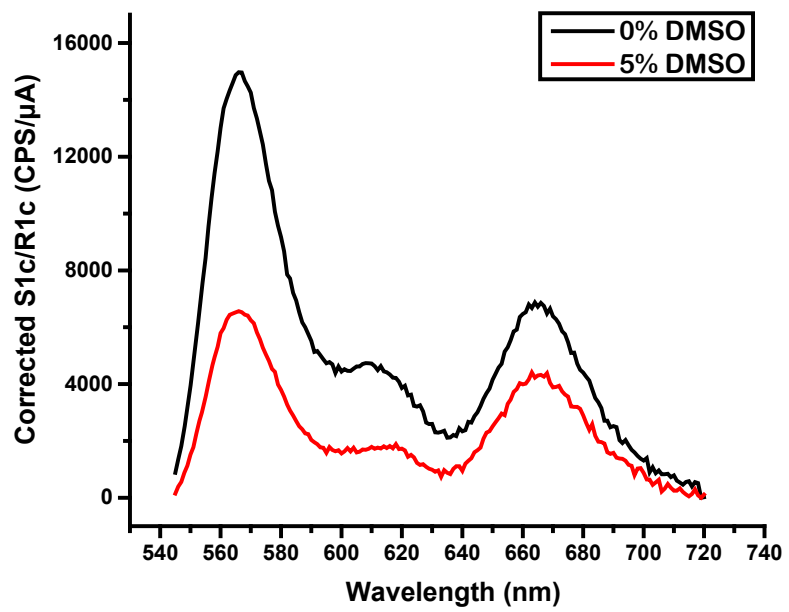

D

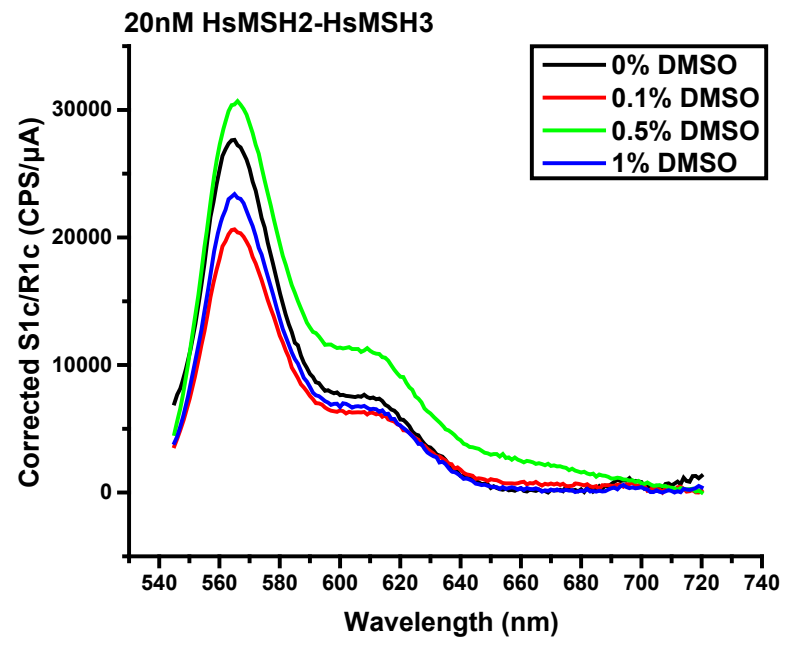


Figure 6

A

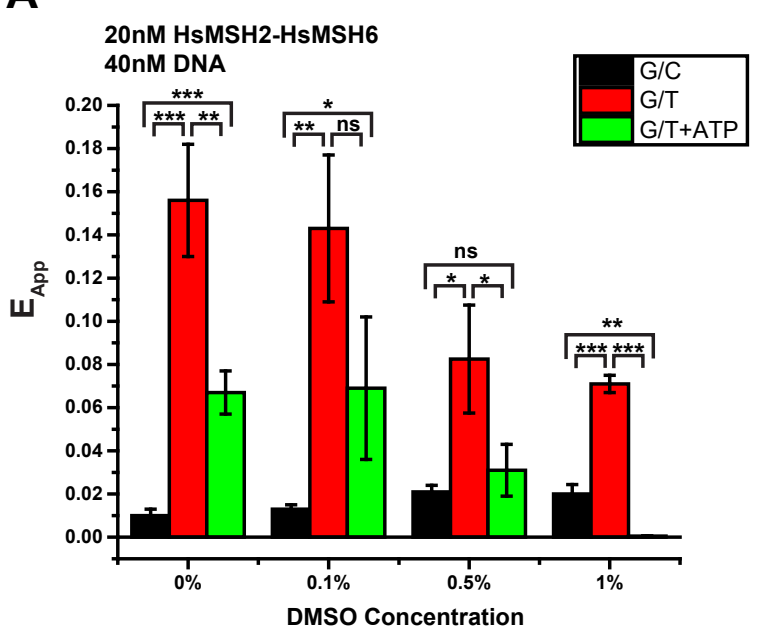

B

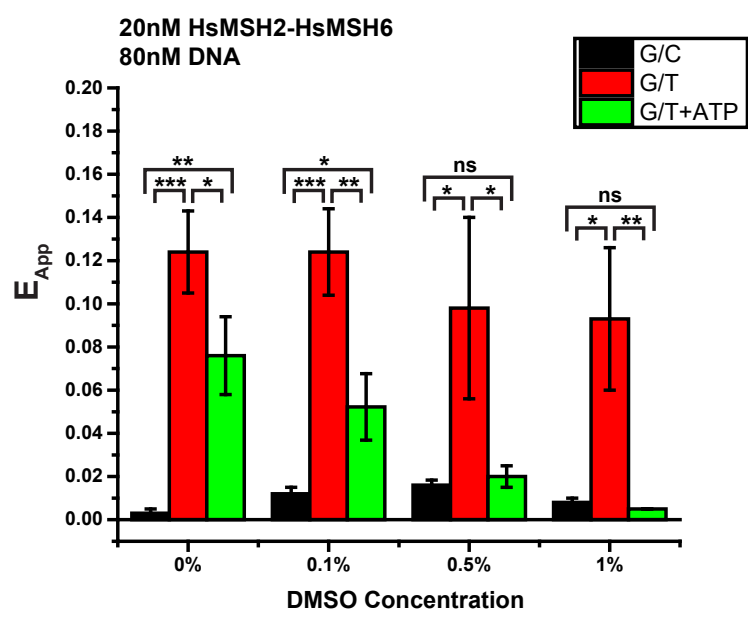


Table 1. Dissociation Kinetics of EcMutS

\begin{tabular}{|c|c|c|c|c|}
\hline \multirow[b]{2}{*}{$\begin{array}{l}\mathrm{NaCl} \\
(\mathrm{mM})\end{array}$} & \multicolumn{2}{|c|}{ Open-End DNA } & \multicolumn{2}{|c|}{ Blocked-End DNA } \\
\hline & $\begin{array}{c}k_{\text {off }}{ }^{a} \\
\left(s^{-1}\right) \times 10^{-2}\end{array}$ & $\begin{array}{c}k_{o f f \bullet A T P}{ }^{b} \\
\left(s^{-1}\right) \times 10^{-2}\end{array}$ & $\begin{array}{c}k_{\text {off }} \\
\left(s^{-1}\right) \times 10^{-2}\end{array}$ & $\begin{array}{c}k_{o f f \cdot A T P} \\
\left(s^{-1}\right) \times 10^{-2}\end{array}$ \\
\hline 15 & $5.7 \pm 0.19$ & $5228 \pm 943$ & $5.9 \pm 0.24$ & $0.53 \pm 0.01$ \\
\hline 30 & $5.9 \pm 0.08$ & $5028 \pm 889$ & $5.8 \pm 0.10$ & $0.58 \pm 0.01$ \\
\hline 60 & $4.8 \pm 0.17$ & $5263 \pm 233$ & $5.4 \pm 0.24$ & $0.59 \pm 0.03$ \\
\hline 80 & $5.1 \pm 0.42$ & $3931 \pm 179$ & $5.5 \pm 0.14$ & $0.67 \pm 0.06$ \\
\hline 100 & $4.8 \pm 0.12$ & $2784 \pm 51$ & $5.3 \pm 0.08$ & $0.66 \pm 0.04$ \\
\hline 120 & $5.0 \pm 0.12$ & $1557 \pm 28$ & $5.7 \pm 0.10$ & $0.86 \pm 0.03$ \\
\hline 140 & $5.1 \pm 0.28$ & $1024 \pm 147$ & $5.5 \pm 0.13$ & $0.85 \pm 0.04$ \\
\hline 160 & $5.4 \pm 0.16$ & $853 \pm 64$ & $6.2 \pm 0.44$ & $0.78 \pm 0.01$ \\
\hline 180 & $5.4 \pm 0.25$ & $481 \pm 69$ & $6.6 \pm 0.26$ & $0.92 \pm 0.04$ \\
\hline
\end{tabular}

${ }^{a}$ First-order rate constants in the absence of ATP $(1 \mathrm{mM})$. \pm Standard deviation of at least three replicates.

${ }^{b}$ First-order rate constants in the presence of ATP $(1 \mathrm{mM})$. \pm Standard deviation of at least three replicates. 


\section{Supporting Table S1}

Name

G-Strand

(Biotin)

T-Strand (Dig)

T-Strand (Open)

Lambda

Mismatch 2A

Lambda

Mismatch 2B

Lambda Hairpin

Linker 1

Lambda Hairpin

Linker 2

Top G-Strand

Bottom T-Strand

Bottom CStrand

Top dT GStrand

Bottom TStrand
Sequence 5' - 3'

/5Biosg/AAAGCTGGAGCTGAAGCTTAGCTTAGGATCATCGAGGA

TCGAGCTCGGTGCAATTCAGCGGTACCCAATTCGCCCTATAGTT

/5DigN/AACTATAGGGCGAATTGGGTACCGCTGAATTGCACCGAG CTTGATCCTCGATGATCCTAAGCTAAGCTTCAGCTCCAGCTTT

AACTATAGGGCGAATTGGGTACCGGTCAATTGCACCGAGCTTG ATCCTCGATGATCCTAAGCTAAGCTTCAGCTCCAGCTTT

Phos-AGGTCGTCGCCCAGAAGATGGGCGAGTTTG

Phos-GCCGCAAACTCGCCCATCTTCT

Phos-

TTCTTGGAGTCCACTGCAGTT/iBiodT/TCTGCAGTGGACTCC

Phos-

CGCTTAGTGCTATGATGCGTT/iBiodT/TCGCATCATAGCACTA

GCTTAGGATCATCGAGGATCGAGCTCGGTGCAATTCAGCGG/3Bi o/

CCGCTGAATTGCACCGAGCTTGATCCTGCATGATCCTAAGC/3Bi o/

CCGCTGAATTGCACCGAGCTCGATCCTCGATGATCCTAAGC/3Bi o/

GCTTAGGATCA/iAmMC6T/CGAGGATCGAGCTCGGTGCAAT TCAGCGG/3Bio/

CCGCTGAATTGCACCGAGCTTGATCCTGCATGATCCTAAGC/ 3Bio/ 


\section{Bottom C- CCGCTGAATTGCACCGAGCTCGATCCTCGATGATCCTAAGC/ Strand 3Bio/}

Bottom +CA4- CCGCTGAATTGCACCGAGCTCACACACACGATCCTCGATGA Strand TCCTAAGC/3Bio/ 


\begin{tabular}{|c|c|c|c|c|}
\hline \multirow[b]{2}{*}{$\begin{array}{l}\mathrm{NaCl} \\
(\mathrm{mM})\end{array}$} & \multicolumn{2}{|c|}{ Open-End DNA } & \multicolumn{2}{|c|}{ Blocked-End DNA } \\
\hline & $\begin{array}{c}k_{\mathrm{off}}{ }^{a} \\
\left(s^{-1}\right) \times 10^{-2}\end{array}$ & $\begin{array}{c}k_{o f f} \cdot A T P^{b} \\
\left(s^{-1}\right) \times 10^{-2}\end{array}$ & $\begin{array}{c}k_{\mathrm{off}} \\
\left(s^{-1}\right) \times 10^{-2}\end{array}$ & $\begin{array}{c}k_{o f f} \cdot A T P \\
\left(s^{-1}\right) \times 10^{-2}\end{array}$ \\
\hline 15 & $0.62 \pm 0.30$ & $20.3 \pm 0.57$ & $1.1 \pm 0.44$ & $0.47 \pm 0.01$ \\
\hline 30 & $0.03 \pm 0.01$ & $24.5 \pm 1.2$ & $0.54 \pm 0.07$ & $0.54 \pm 0.02$ \\
\hline 80 & $0.88 \pm 0.12$ & $26.7 \pm 0.77$ & $1.4 \pm 0.15$ & $0.35 \pm 0.01$ \\
\hline 100 & $0.61 \pm 0.02$ & $26.6 \pm 0.93$ & $1.6 \pm 0.09$ & $0.56 \pm 0.00$ \\
\hline 120 & $0.84 \pm 0.07$ & $28.8 \pm 1.2$ & $2.2 \pm 0.13$ & $0.55 \pm 0.00$ \\
\hline 140 & $1.3 \pm 0.09$ & $30.3 \pm 1.5$ & $2.8 \pm 0.80$ & $0.52 \pm 0.01$ \\
\hline 160 & $1.2 \pm 0.12$ & $23.6 \pm 1.3$ & $4.6 \pm 0.88$ & $0.52 \pm 0.01$ \\
\hline 180 & $0.92 \pm 0.05$ & $19.0 \pm 0.68$ & $6.3 \pm 1.3$ & $0.60 \pm 0.01$ \\
\hline
\end{tabular}

${ }^{a}$ First-order rate constants in the absence of ATP $(1 \mathrm{mM})$. \pm Standard deviation of at least three replicates.

${ }^{b}$ First-order rate constants in the presence of ATP $(1 \mathrm{mM})$.

\pm Standard deviation of at least three replicates.

Supporting Table 2. Dissociation Kinetics of HsMSH2-HsMSH6. Surface plasmon resonance dissociation studies. First-order rate constants of HsMSH2-HsMSH6 (40 nM) in the absence ( $\left.\mathrm{k}_{\text {off }}\right)$

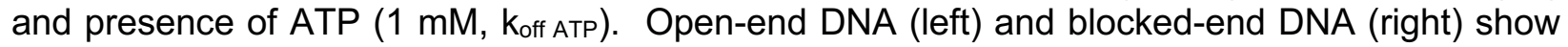
similar rates of dissociation in the absence of ATP $\left(\mathrm{K}_{\text {off }}\right)$. HsMSH2-HsMSH6 sliding clamps quickly slide off open-end DNA (left) but are retained for $\sim 3$ minutes on blocked-end DNAs (right). 


\section{Supporting Figure S1}

A

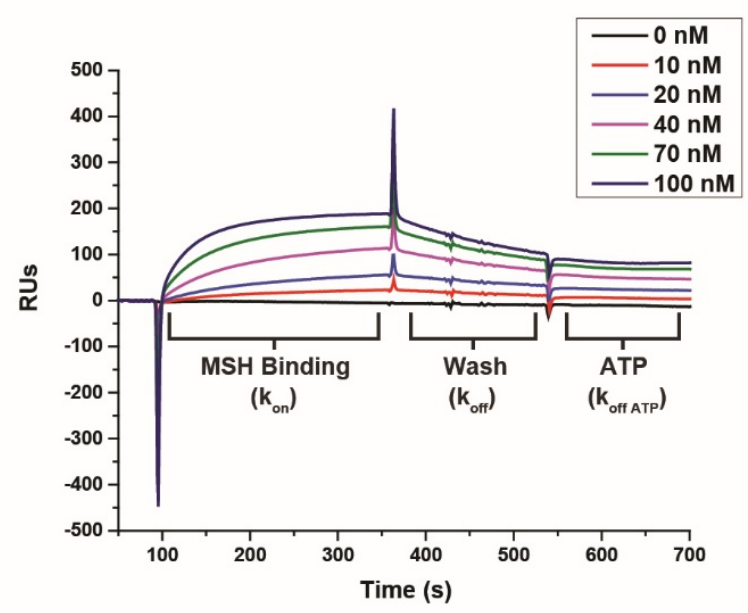

B

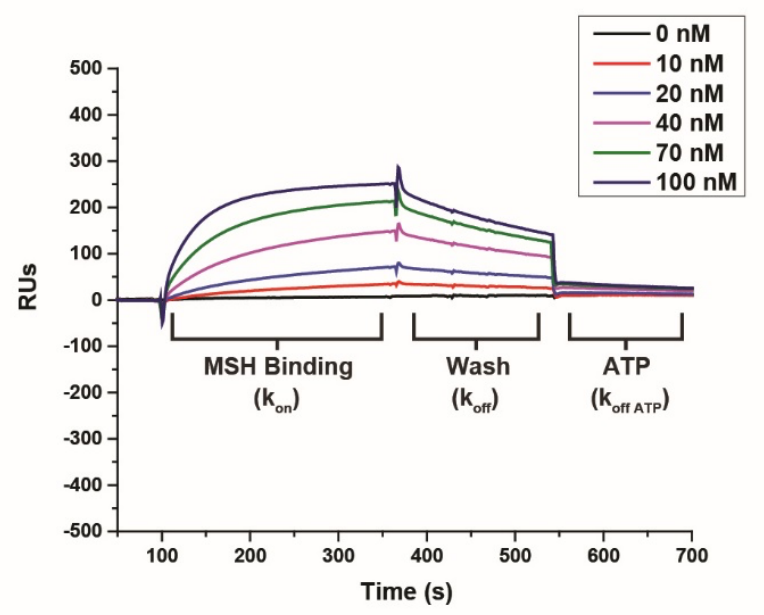

Supporting Figure S1. EcMutS Binding Analysis. Surface plasmon resonance association studies. (A) Protein titration of EcMutS on blocked-end DNA. (B) Protein titration of EcMutS on open-end DNA. MSH Binding indicates where MSH (40 nM) protein is flowed onto chip. This region is used to calculate the second-order rate constant $\left(k_{o n}\right)$. Wash indicates where the unbound protein is washed from chip. This region is used to calculate the first-order rate constant $\left(k_{\text {off }}\right)$. ATP indicates where ATP $(1 \mathrm{mM})$ in buffer is flowed onto chip. This region is used to calculate the first-order rate constant $\left(k_{\text {off-ATP }}\right)$. 


\section{Supporting Figure S2}

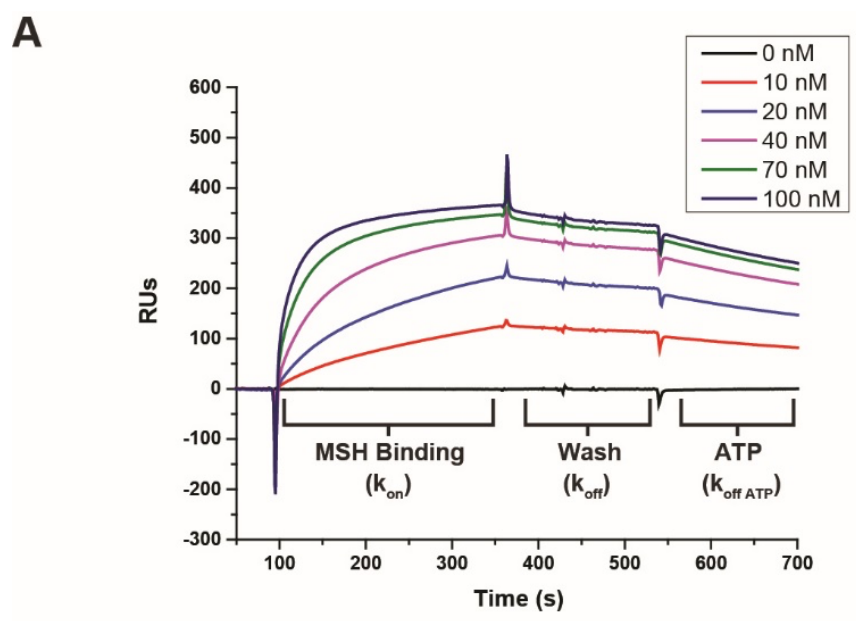

B

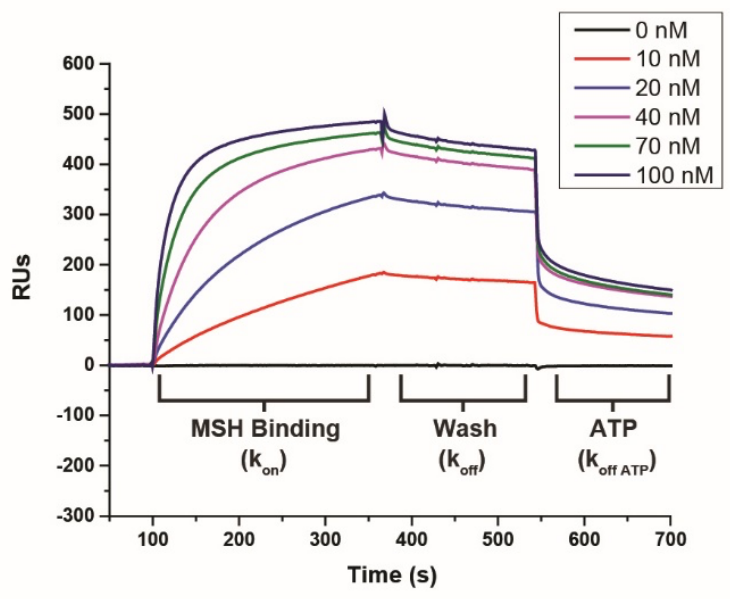

Supporting Figure S2. HsMSH2-MSH6 Binding Analysis. Surface plasmon resonance association studies. (A) Protein titration of HsMSH2-MSH6 on blocked-end DNA. (B) Protein titration of HsMSH2-MSH6 on open-end DNA. MSH Binding indicates where MSH (40 nM) protein is flowed onto chip. This region is used to calculate the second-order rate constant $\left(k_{\text {on }}\right)$. Wash indicates where the unbound protein is washed from chip. This region is used to calculate the first-order rate constant $\left(k_{\text {off }}\right)$. ATP indicates where ATP $(1 \mathrm{mM})$ in buffer is flowed onto chip. This region is used to calculate the first-order rate constant $\left(k_{\text {off.ATP }}\right)$. 


\section{Supporting Figure S3}
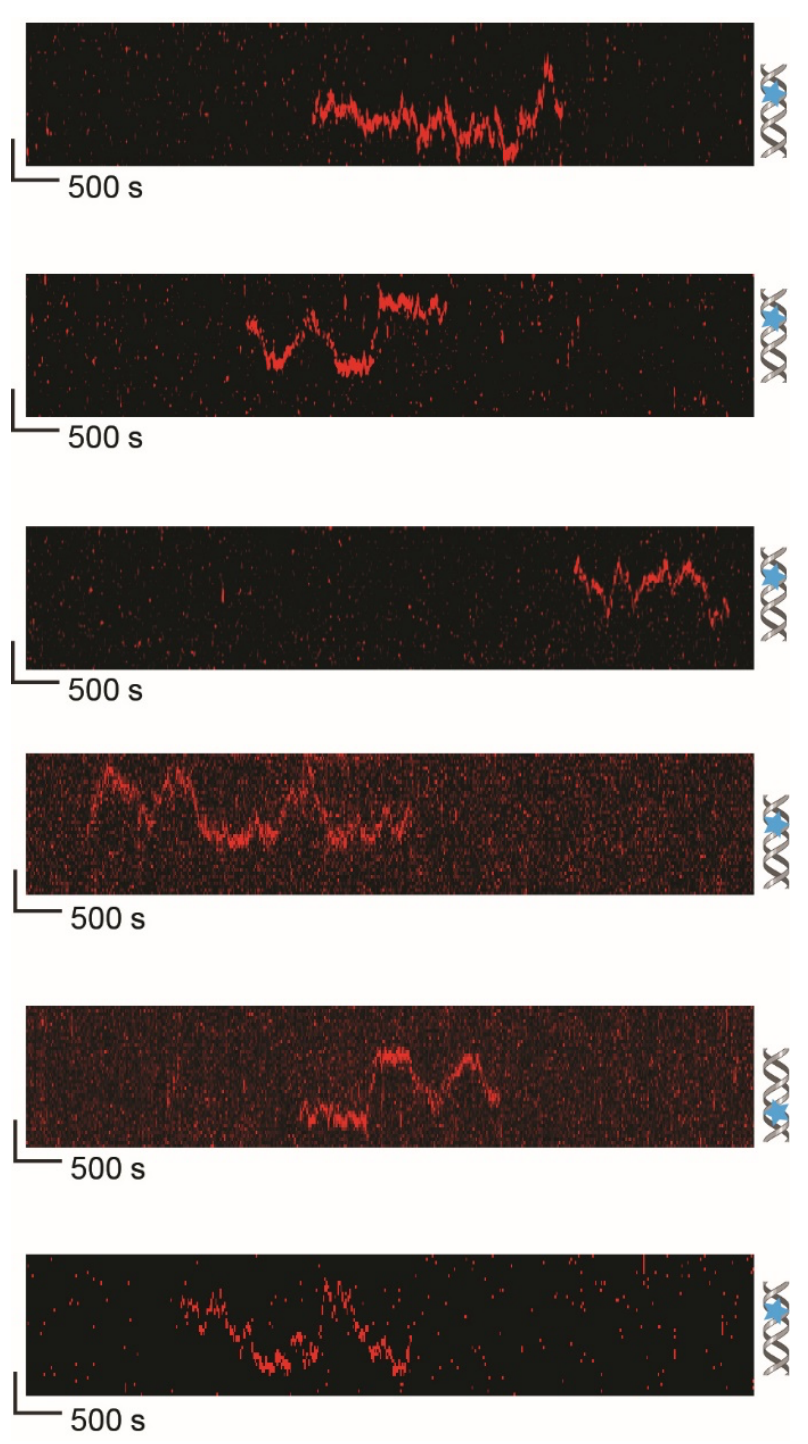

Supporting Figure S3. Additional MutS Kymographs. Representative kymographs of EcMutS DNA search. Blue star indicates the mismatch position. 


\section{Supporting Figure S4}
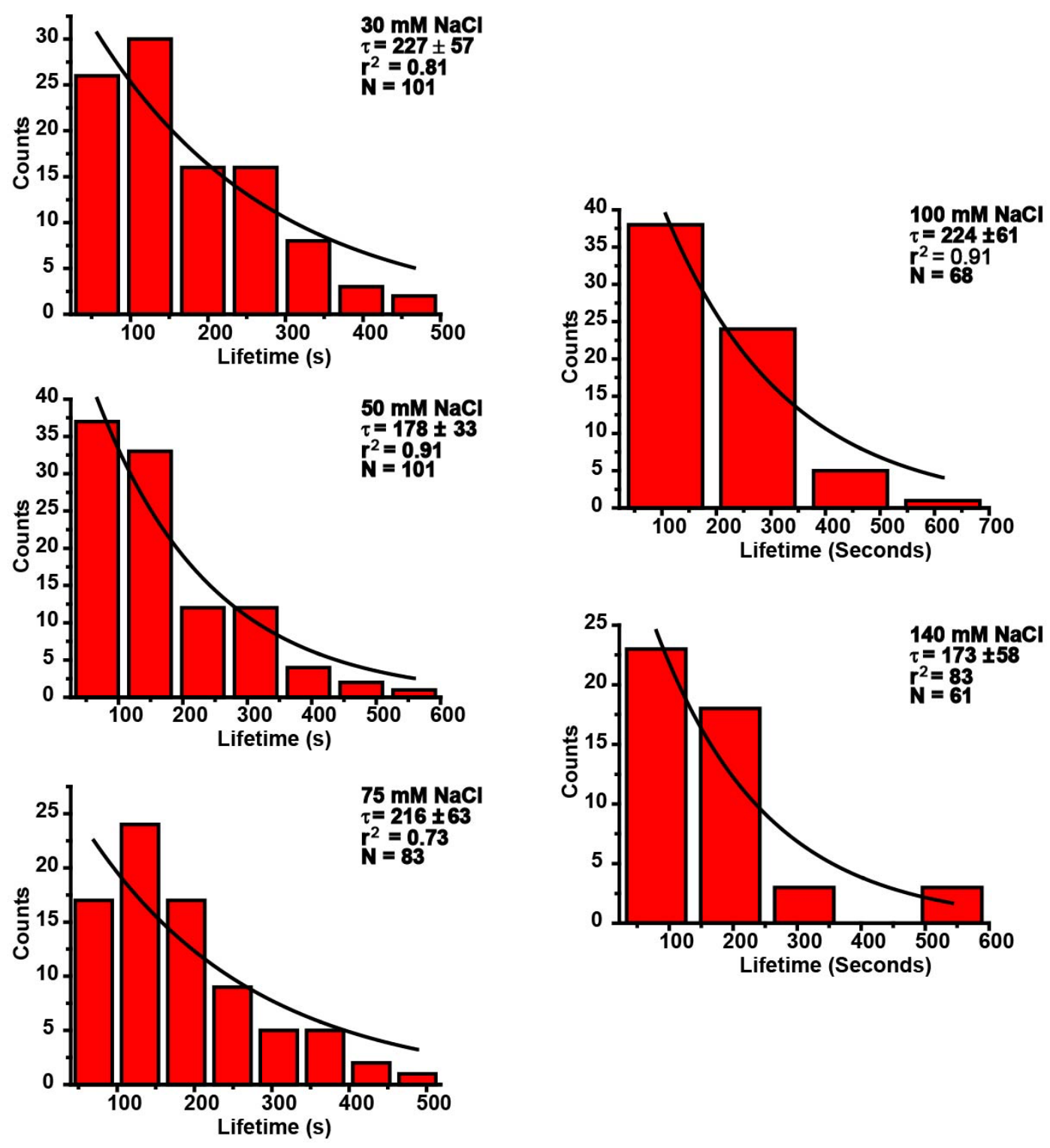

Supporting Figure S4. MutS Lifetime Calculations. Distribution of lifetime of EcMutS on a single mismatched DNA at various ionic strengths as indicated. 


\section{Supporting Figure S5}

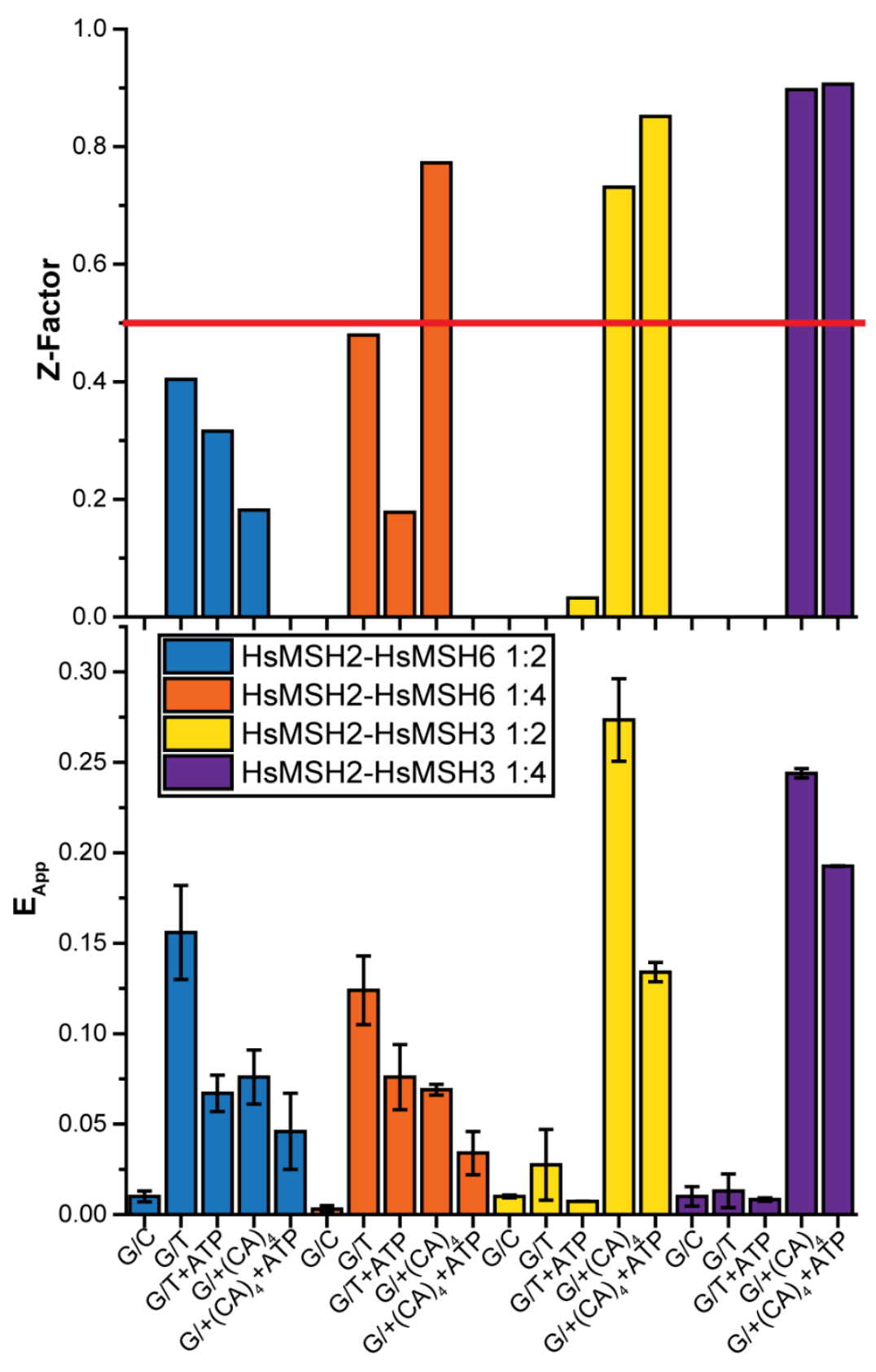

Supporting Figure S5. Z-Factor. (Top) Z-Factor quantification of assay effectiveness. Only values greater than zero shown. Red line shows 0.5 which indicates a high-quality drug screen. (Bottom) Composite $E_{\text {App }}$ bar graph used to calculate Z-factor of 1:2 (HsMSH2-HsMSH6:DNA), 1:4 (HsMSH2-HsMSH6:DNA), 1:2 (HsMSH2-HsMSH3:DNA), 1:4 (HsMSH2-HsMSH3:DNA) for $\mathrm{G} / \mathrm{C} ; \mathrm{G} / \mathrm{T} ; \mathrm{G} / \mathrm{T}+\mathrm{ATP} ; \mathrm{G} /+(\mathrm{CA})_{4} ; \mathrm{G} /+(\mathrm{CA})_{4}+\mathrm{ATP}$ (mean $\pm \mathrm{SD}$ ). 